\title{
Metabolic reprogramming ensures cancer cell survival despite oncogenic signaling blockade
}

\author{
Hui-wen Lue, ${ }^{1}$ Jennifer Podolak, ${ }^{1}$ Kevin Kolahi, ${ }^{2}$ Larry Cheng, ${ }^{3}$ Soumya Rao, ${ }^{1}$ Devin Garg, ${ }^{1}$ \\ Chang-Hui Xue, ${ }^{1}$ Juha K. Rantala, ${ }^{1}$ Jeffrey W. Tyner, ${ }^{1}$ Kent L. Thornburg, ${ }^{2}$ Ann Martinez-Acevedo, ${ }^{4}$ \\ Jen-Jane Liu, ${ }^{4}$ Christopher L. Amling, ${ }^{4}$ Charles Truillet, ${ }^{5}$ Sharon M. Louie, ${ }^{6}$ Kimberly E. Anderson, ${ }^{6}$ \\ Michael J. Evans, ${ }^{5}$ Valerie B. O'Donnell, ${ }^{7}$ Daniel K. Nomura, ${ }^{6}$ Justin M. Drake, ${ }^{3}$ Anna Ritz, ${ }^{8}$ \\ and George V. Thomas ${ }^{1,9}$ \\ ${ }^{1}$ Knight Comprehensive Cancer Institute, Oregon Health and Science University, Portland, Oregon 97239, USA; ${ }^{2} \mathrm{Knight}$ \\ Cardiovascular Institute, Oregon Health and Science University, Portland, Oregon 97239, USA; ${ }^{3}$ Rutgers Cancer Institute of New \\ Jersey, Rutgers, The State University of New Jersey, New Brunswick, New Jersey 08903, USA; ${ }^{4}$ Department of Urology, Oregon \\ Health and Science University, Portland, Oregon 97239, USA; ${ }^{5}$ Department of Radiology, University of California at San Francisco \\ School of Medicine, San Francisco, California 94107, USA; ${ }^{6}$ University of California at Berkeley, Berkeley, California 94720, USA; \\ ${ }^{7}$ Systems Immunity Research Institute, Cardiff University, Cardiff CF14 4XN, United Kingdom; ${ }^{8}$ Department of Biology, Reed \\ College, Portland, Oregon 97202, USA; ${ }^{9}$ Department of Pathology and Laboratory Medicine, Oregon Health and Science \\ University, Portland, Oregon 97239, USA
}

There is limited knowledge about the metabolic reprogramming induced by cancer therapies and how this contributes to therapeutic resistance. Here we show that although inhibition of PI3K-AKT-mTOR signaling markedly decreased glycolysis and restrained tumor growth, these signaling and metabolic restrictions triggered autophagy, which supplied the metabolites required for the maintenance of mitochondrial respiration and redox homeostasis. Specifically, we found that survival of cancer cells was critically dependent on phospholipase A2 (PLA2) to mobilize lysophospholipids and free fatty acids to sustain fatty acid oxidation and oxidative phosphorylation. Consistent with this, we observed significantly increased lipid droplets, with subsequent mobilization to mitochondria. These changes were abrogated in cells deficient for the essential autophagy gene ATG5. Accordingly, inhibition of PLA2 significantly decreased lipid droplets, decreased oxidative phosphorylation, and increased apoptosis. Together, these results describe how treatment-induced autophagy provides nutrients for cancer cell survival and identifies novel cotreatment strategies to override this survival advantage.

[Keywords: autophagy; cancer; metabolism; phospholipid; resistance; signaling]

Supplemental material is available for this article.

Received July 25, 2017; revised version accepted October 26, 2017.

Despite significant advances in precision cancer therapies, tumor regressions are variable and rarely complete. Although the molecular basis of how cancer cells survive therapies that are designed to kill them (i.e. drug-tolerant "persister" cells) is likely due to a mixed set of mechanisms, we reasoned that at its root are subpopulations of drug-tolerant cancer cells that can rewire their signaling and metabolic networks to adapt to treatment-imposed proliferative, survival, and nutrient restrictions. While rewired compensatory oncogenic signaling (e.g., mediated through bypass pathways, receptor amplification, and second site mutations) have been well documented, little is known about the metabolic reprogramming induced by treatment and how this contributes to resistance.

To better understand the metabolic consequences of anti-cancer treatment, we studied metabolic reprogram-

Corresponding author: thomasge@ohsu.edu

Article published online ahead of print. Article and publication date are online at http://www.genesdev.org/cgi/doi/10.1101/gad.305292.117. ming in the context of PI3K pathway inhibition. The PI3K pathway, which includes the PI3K holoenzyme and its truncal effector kinases, AKT and mTOR, is essential for cell growth, proliferation, survival, and metabolism. However, clinical responses to PI3K-AKT-mTOR inhibitors have been modest to date (Fruman and Rommel 2014; Toska and Baselga 2016). We hypothesized that the limited ability of PI3K-AKT-mTOR inhibitors to induce cancer cell death was due to the autophagy-mediated metabolic reprogramming that enabled drug-tolerant cells to survive despite therapy-enforced nutrient restrictions. Our hypothesis was based on the knowledge that nutrients derived from autophagic degradation are reused to maintain macromolecular synthesis and or oxidized to

(C) 2017 Lue et al. This article is distributed exclusively by Cold Spring Harbor Laboratory Press for the first six months after the full-issue publication date (see http://genesdev.cshlp.org/site/misc/terms.xhtml). After six months, it is available under a Creative Commons License (Attribution-NonCommercial 4.0 International), as described at http://creativecommons.org/licenses/by-nc/4.0/. 
maintain bioenergetics (Galluzzi et al. 2015). Additionally, due to the central role that the PI3K-AKT-mTOR pathway has in regulating cellular growth, we reasoned that small molecule inhibitors that converge directly or indirectly on this pathway would similarly induce autophagy to sustain drug-tolerant cells, therefore extending the reach of this mechanism of resistance beyond specific PI3K-AKT-mTOR inhibitors.

Thus far, the therapeutic reflex to block autophagy is to add anti-malarial lysosomotropic inhibitors such as chloroquine, but the clinical responses to these drugs have been variable and noncurative (Goldberg et al. 2012; Shanware et al. 2013; Rosenfeld et al. 2014; Towers and Thorburn 2016). Therefore, it would be clinically impactful to directly target the metabolic enzymes mediating autophagy-fueled metabolic processes on which drug-tolerant cells are dependent. However, there have not been any therapeutically tractable metabolic enzymes identified in the setting of therapy-induced autophagy.

Here, we identify CYT387, a JAK inhibitor that induces autophagy by inhibiting mTOR complex 1 (mTORC1). Consequently, by relieving the inhibitory signal transmitted from mTORC1 to PI3K, treatment with CYT387 leads to activation of the PI3K-mTORC2/AKT pathway. Combining CYT387 with MK2206, an allosteric AKT inhibitor, did not induce any tumor regressions despite effectively inhibiting PI3K-AKT-mTORC1/2 activation. Notably, the combination treatment further increased autophagy. This suggested that inhibition of signaling pathways alone would be insufficient to kill all tumor cells. Subsequently, we performed global metabolic profiling to systematically document the immediate metabolic adaptations effected by the therapy-induced autophagic processes. We show that autophagy-mediated metabolic adaptations supported cancer cell survival. Autophagy was required for these metabolic adaptations because these changes were abrogated in cells deficient for the essential autophagy gene ATG5. Subsequently, we identified that phospholipase A2 (PLA2), the rate-limiting enzyme responsible for catalyzing the breakdown of phospholipids to lysophospholipids and fatty acids, had an important role in the survival of cancer cells. Pharmacological inhibition of this enzyme dampened oxidative phosphorylation (OXPHOS) and further increased apoptosis when combined with CYT387MK2206 combination treatment. Our findings highlight a previously unappreciated role for PLA2 in conferring a survival advantage to drug-tolerant cancer cells in metabolically restricted environments, demonstrate that this enzyme supports autophagy-induced metabolic reprogramming, and, importantly, provide a path forward for novel cotreatment strategies.

\section{Results}

CYT387 induces autophagy through the modulation of the PI3K-AKT-mTOR pathway

To precisely identify which cancer drugs induce autophagic flux by inhibiting the mTORC1 pathway, we used a library of 116 clinically focused and mechanistically annotated compounds that included activity against two-thirds of the tyrosine kinome as well as other nontyrosine kinase pathways on a human renal cell carcinoma (RCC) cell line, ACHN (Leonard et al. 2016; Maxson et al. 2013, 2016) (see Supplemental Fig. S1A for a schematic of the workflow; see Supplemental Table 1 for a list of drugs and known targets). We monitored mTORC1 activity through phosphorylation of S6 and combined this with a measurement of $\mathrm{p} 62$ steady-state levels as an initial screen of autophagy flux (Joachim et al. 2015) in a highcontent imaging screen.

Remarkably, the screen identified several structurally different Janus kinase (JAK) inhibitors as potent inducers of autophagic flux; namely, pan-Jak inhibitor (JAK1, JAK2, and JAK3), Go6978 (JAK 2), ruxolitinib (Jak1 and Jak2), and CYT387 (JAK1 and JAK2). All four drugs potently inhibited S6 phosphorylation, pointing to a mTORC1dependent mechanism. Since JAK inhibitors as a class of compounds scored highly in our screen and because CYT387 was the most potent JAK inhibitor to induce autophagic flux and simultaneously decrease S6 phosphorylation in solid tumor cells in our screen, we selected this small molecule for further validation. CYT387 (momelutinib) is an orally available JAK1-2 inhibitor that has improved splenomegaly and reduced anemia in myeloproliferative neoplasia (MPN) patients (Patel et al. 2016; Winton and Kota 2017). In support of this, CYT387 suppressed the phosphorylation of JAK; its substrate, STAT3; and S6 in human RCC and MPN cell lines (Supplemental Fig. S1B,C). CYT387 induces autophagy that is reversible-as seen by the reduction in LC3B lipidation within $24 \mathrm{~h}$ of removal of drug - and correlated with reversal of the p-STAT3, p-S6, and p-AKT phosphorylation patterns (Supplemental Fig. S1D).

CYT387 treatment of ACHN human RCC cells plated on coverslips resulted in decreased $\mathrm{p} 62$ protein expression and phosphorylated S6 levels by immunofluorescence staining, confirming our high-content imaging finding (Fig. 1A). Accordingly, we observed that treatment with CYT387 induced autophagy in multiple human RCC and MPN cell lines and was primarily cytostatic (Supplemental Fig. S1E,F). Immunoblots confirmed the induction of autophagy by CYT387, as seen by the conversion of LC3-I to LC3-II, the degradation of p62, and inhibition of mTORC1 (as seen by decrease in phosphorylated S6) (Fig. 1B). We additionally confirmed that CYT387 treatment induced autophagic flux by several different methods. (1) We stably expressed a mChery-EGFP-LC3 reported in ACHN cells, which takes advantage of the fact that EGFP fluorescence is quenched in the acidic environment of the autolysosome relative to mCherry (Debnath 2008). CYT387 treatment resulted in decreased expression of green-yellow cells and increased expression of red cells (Supplemental Fig. S2A). (2) We stained CYT387-treated ACHN cells with the autofluorescent compound monodansylcadaverine (MDC), a marker of autolysosomes, and found that CYT387 increased MDC autofluorescence (Supplemental Fig. S2B; Turcotte et al. 2008). (3) CYT387 increased LC3-II levels in ACHN cells, and this increase was more pronounced in the presence of 
A

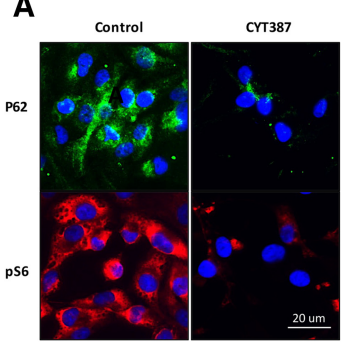

B

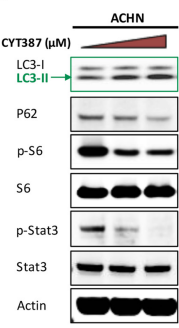

E

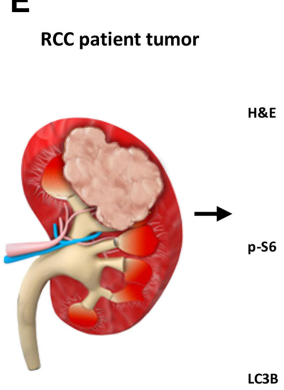

C

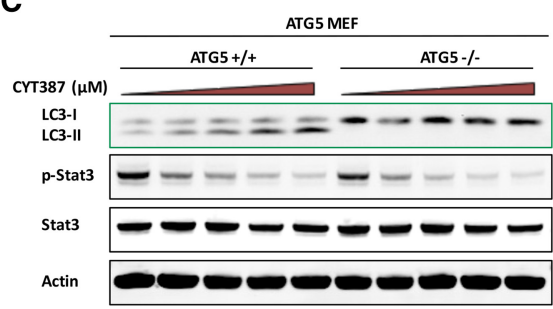

F

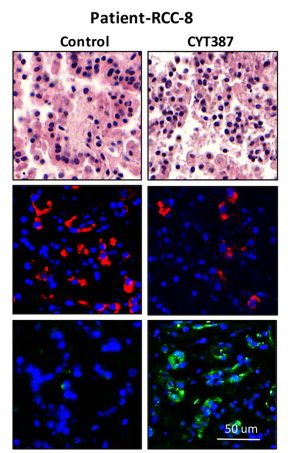

D

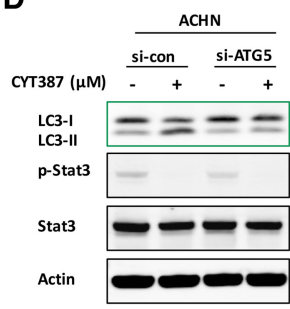

G
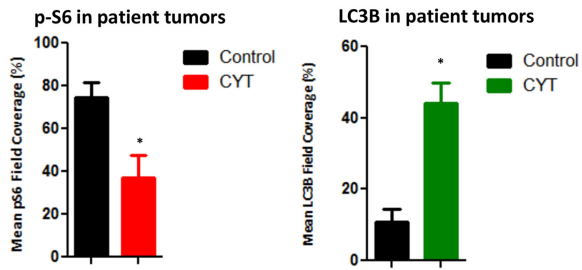

H
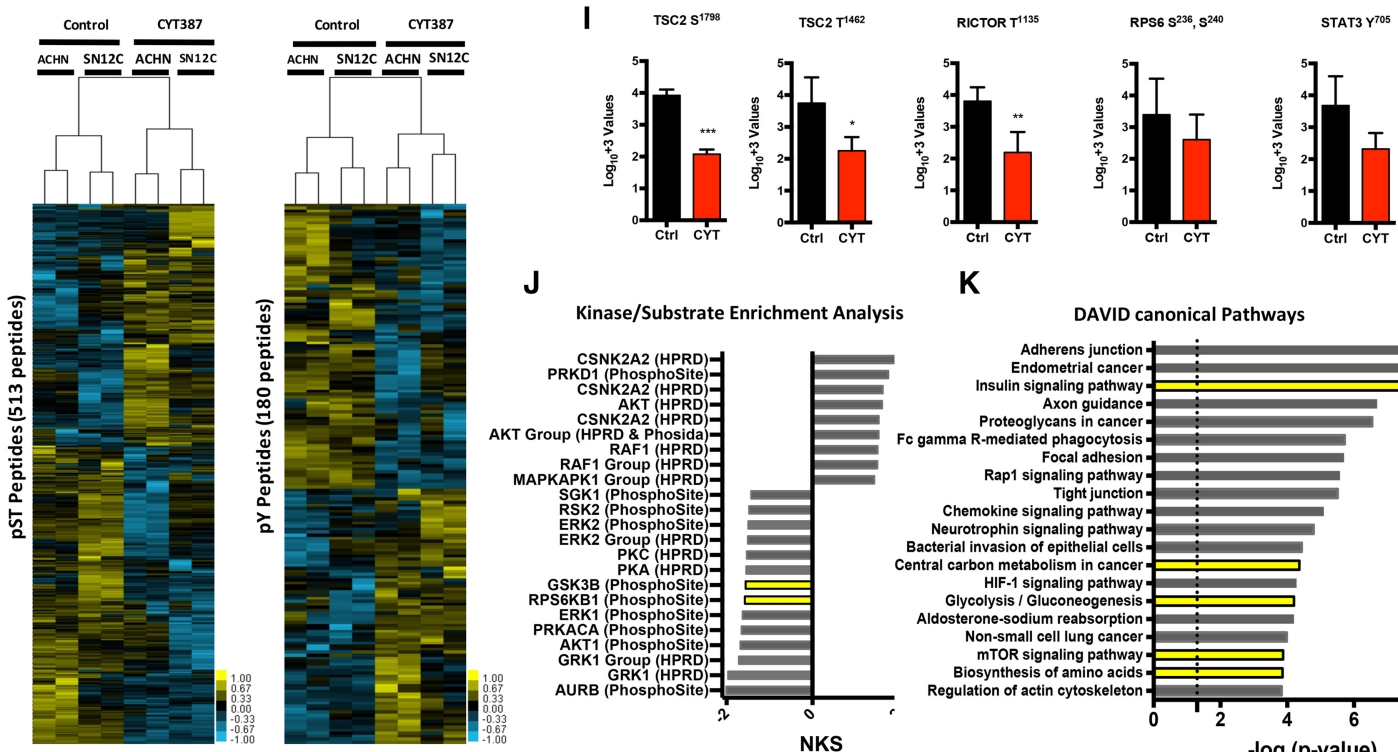

$\mathbf{J}$

K
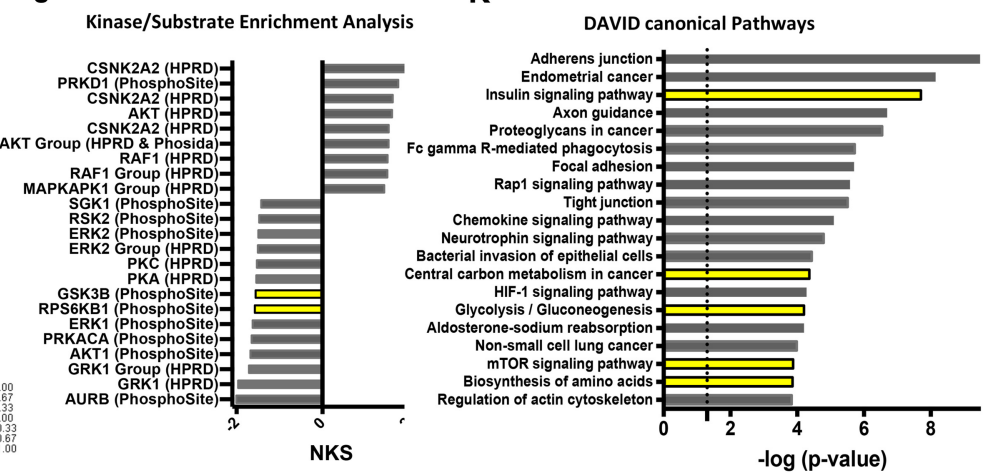

Figure 1. CYT387 induces autophagy in human cancer cell lines and patient-derived models. (A) ACHN cells were grown on coverslips, treated with CYT387 for $24 \mathrm{~h}$, and stained p62 and p-S6. (B) ACHN cells were treated with increasing doses of CYT387 (0-2 $\mu$ M) and immunoblotted with LC3, p62, p-S6, total S6, p-STAT3, total STAT3, and $\beta$-actin. $(C) A T G 5^{+/+}$and ATG5 ${ }^{-/-}$MEFs were treated with 0-3 $\mu \mathrm{M}$ CYT387 for $24 \mathrm{~h}$, and LC3 processing was evaluated by immunoblotting. (D) Immunoblot for LC3 and p-STAT3 and ATG5 in ACHN cells transiently transfected with siRNA against ATG5. $\beta$-Actin was used as a loading control. (E) Patient-derived organotypic cultures were treated with CYT387 for $24 \mathrm{~h}$ and stained with p-S6 and LC3B (images from two different patient tumors are shown). (F, $G$ ) Quantification of p-S6 $(F)$ and LC3B $(G)$ staining in patient-derived organotypic cultures (bar graph). $n=10$ patients. $(H)$ Supervised hierarchical clustering heat maps of phosphoserine and phosphothreonine (pST) peptides and phosphotyrosine (pY) peptides identified from CYT387-treated and untreated ACHN and SN12C human RCC cells with two technical replicates. Five-hundred-thirteen unique pST phosphopeptides (rows) and 180 unique pY phosphopeptides were either fourfold more enriched or fourfold less enriched, on average ( $t$-test, $P<0.2)$, in CYT387-treated cells compared with untreated cells (phosphopeptide lists are in Supplemental Tables 2, 3). $(I)$ Relative phosphorylation abundance between ACHN and SN12C CYT387-treated and untreated cells. RPS6 S ${ }^{236}, S^{240}$ was found in the pST unsupervised heat map. (Ctrl) Control; (CYT) CYT387. $\left({ }^{*}\right) P<0.05 ;\left({ }^{* *}\right) P<0.01 ;\left({ }^{* * *}\right) P<0.001$, unpaired $t$-test. $(J)$ Kinase substrate enrichment analysis (KSEA) of CYT387-treated and untreated pST data. Hits $>5$; false discovery rate $[\mathrm{FDR}]<0.05$. A positive NKS (normalized Kolmogorov-Smirnov score) infers greater kinase activity in CYT387-treated cells, while a negative NKS indicates greater activity in untreated cells (unfiltered summary is in Supplemental Table 4). (K) KEGG (Kyoto Encyclopedia of Genes and Genomes) pathways found from DAVID analysis of relatively active genes due to CYT387 treatment. The DAVID input list was generated by interpreting functional annotations of pSTY data (the complete pathway list is in Supplemental Table 2). 
E64D/pepstatin /which inhibits the protease-induced reconversion of LC3-II into LC3-I), consistent with an increase in autophagosome formation (Supplemental Fig. S2C; Tanida et al. 2005). (4) CYT387 increased the number of double-membraned autophagosomes, which are pathognomonic of autophagy as determined by transmission electron microscopy (Supplemental Fig. S2D; Klionsky et al. 2016). Notably, CYT387 was able to induce autophagy in a dose-dependent manner in murine embryonic fibroblasts (MEFs) that retained the essential autophagy gene ATG5 $\left(A T G 5^{+/+}\right)$, as seen by the lipidation of LC3 (Fig. 1C) (Cecconi and Levine 2008; Fung et al. 2008). Conversely, CYT387 did not induce autophagy in ATG5-deficient cells $\left(A T G 5^{-1-}\right)$. Likewise, CYT387-induced autophagy was abrogated with siRNA depletion of ATG5 in ACHN cells (Fig. 1D). To extend our studies into clinical samples, we exposed patient-derived RCC organotypic cultures to CYT387 treatment for 24 h. Importantly, CYT387 significantly induced LCB expression while simultaneously reducing phosphorylated S6 levels (Fig. 1E,F). Taken together, these results indicate that CYT387 treatment induces autophagic flux in both human RCC cell lines and patient-derived tumors.

To obtain further insight into the signaling pathways affected by CYT387 treatment, we studied changes in the phosphoproteome of two different human RCC cells (ACHN and SN12C) after CYT387 treatment using quantitative phosphoproteomics (Rush et al. 2005; Moritz et al. 2010; Zhuang et al. 2013). Supervised hierarchical clustering revealed that 513 phosphoserine and phosphothreonine (pST) peptides and 180 phosphotyrosine (pY) peptides significantly differed between treated and untreated cells (Fig. 1H; Supplemental Tables 2-9). We observed two phosphopeptides to be hypophosphorylated at inhibitory residues T1462 and S1798 in tuberous sclerosis complex 2 (TSC2) in CYT387-treated cells (Manning et al. 2002; Roux et al. 2004). Rapamycin-insensitive companion of mTOR (RICTOR) in CYT387-treated cells was hypophosphorylated at T1135. RICTOR is a subunit of mTORC2 (Kim et al. 2017), but the phosphorylation of T1135 is mediated by mTORC1 via induction of the p70S6 kinase (Julien et al. 2010) and impedes the ability of mTORC2 to phosphorylate AKT on S473 (Fig. 1I; Dibble et al. 2009). As expected, ribosomal protein S6 at residues S236 and S240 and STAT3 Y705 trended toward hypophosphorylation, and p70S6 kinase (RPS6KB) was significantly less active in CYT387-treated cells based on kinase substrate enrichment analyses (KSEAs) (Fig. 1J; Drake et al. 2012). However, KSEAs of AKT motifs were inconclusive, as some motifs trended toward increased activity and others trended toward decreased activity in CYT387-treated cells. DAVID analysis of genes corresponding to the phosphopeptides and activated in CYT387-treated cells (Supplemental Tables 10, 11) also revealed several KEGG (Kyoto Encyclopedia of Genes and Genomes) pathways that are biologically relevant to CYT387 treatment, including glycolysis, amino acid biosynthesis, and central carbon metabolism (Fig. 1K; Huang da et al. 2009a,b). In support of these phosphoproteomics findings, mRNA analysis of CYT387-treated ACHN cells using gene set enrichment analysis (GSEA) of multiple independent data sets revealed significant enrichment of genes involved in several metabolic pathways, while biological modules associated with $\mathrm{mTOR}$ (e.g., cell cycle and protein synthesis) were anti-correlated with CYT387 treatment. (Supplemental Tables 12, 13).

Collectively, the phosphoproteome and transcriptome data provide strong evidence that CYT387 treatment reduces mTORC1 signaling to increase TSC2 and mTORC2 signaling leading to AKT activation and is coupled with changes in metabolic pathways.

\section{PI3K-AKT-mTOR inhibition treatment restrains tumor growth but does not induce tumor regression}

We reasoned that the CYT387-induced inhibition of mTORC1 would relieve the inhibitory feedback signal normally transmitted from mTORC1 to PI3K, as the phosphoproteomic data suggested via KSEA, and that this would result in hyperactivation of $\mathrm{PI} 3 \mathrm{~K}$ and $\mathrm{AKT}$, with consequent prosurvival signaling. Consistent with this interpretation, CYT387 treatment caused an increase in AKT T308, the PDK-1-catalyzed site that serves as readout for PI3K signaling in a time-dependent manner (Supplemental Fig. S3A,B). Notably, CYT387 did not dephosphorylate ERK (Supplemental Fig. S3C). Therefore, we sought to identify PI3K-AKT pathway inhibitors that would effectively cooperate with CYT387 to induce apoptosis. We used GDC-0941, a pan-PI3K inhibitor (Sarker et al. 2015); BX795, a PDK-1 inhibitor (Dangelmaier et al. 2014); and MK2206 (Yap et al. 2011), an allosteric AKT inhibitor, to chemically deconstruct this signaling pathway, as depicted in the schematic (Supplemental Fig. S3D-F). We first assessed the biologic effects of these inhibitors on proliferation and apoptosis in human RCC cells singly and in combination with CYT387 (Fig. 2AD). While GDC-0941, BX795, and MK2206 alone exhibited some anti-proliferative effects, the combination with CYT387 resulted in significantly greater inhibition of proliferation in ACHN and SN12C cells. In marked contrast, all drugs as single agents had little or no effect on apoptosis, but the combination of either agent with CYT387 resulted in increased apoptosis. This was most striking in the CYT387 and MK2206 combination (Fig. 2B,D), and we therefore selected MK2206 for further in vivo studies. We investigated the mechanisms by which MK2206 and CYT387 cooperated to suppress tumor growth in RCC cells (Fig. 2E,F). MK2206 effectively inhibited AKT activation, as documented by dephosphorylation of both p-AKT Thr308 and p-Ser473 and the AKT substrate PRAS40. Consistent with prior results, suppression of AKT induced autophagy, as seen by the conversion of LC3-I to LC3-II. Suppression of mTORC1 by CYT387 led to feedback activation of $\mathrm{PI} 3 \mathrm{~K}$, as seen by the increase in phosphorylation of p-AKT Thr308 (which serves as a readout for PI3K activity) and mTORC2 (as monitored by AKT Ser473 phosphorylation). Subsequently, combining MK2206 with CYT387 effectively inhibited both AKT and mTORC1 to almost undetectable levels and induced apoptosis (cleaved caspase 3). Thus, by inhibiting the PI3K-AKT- 

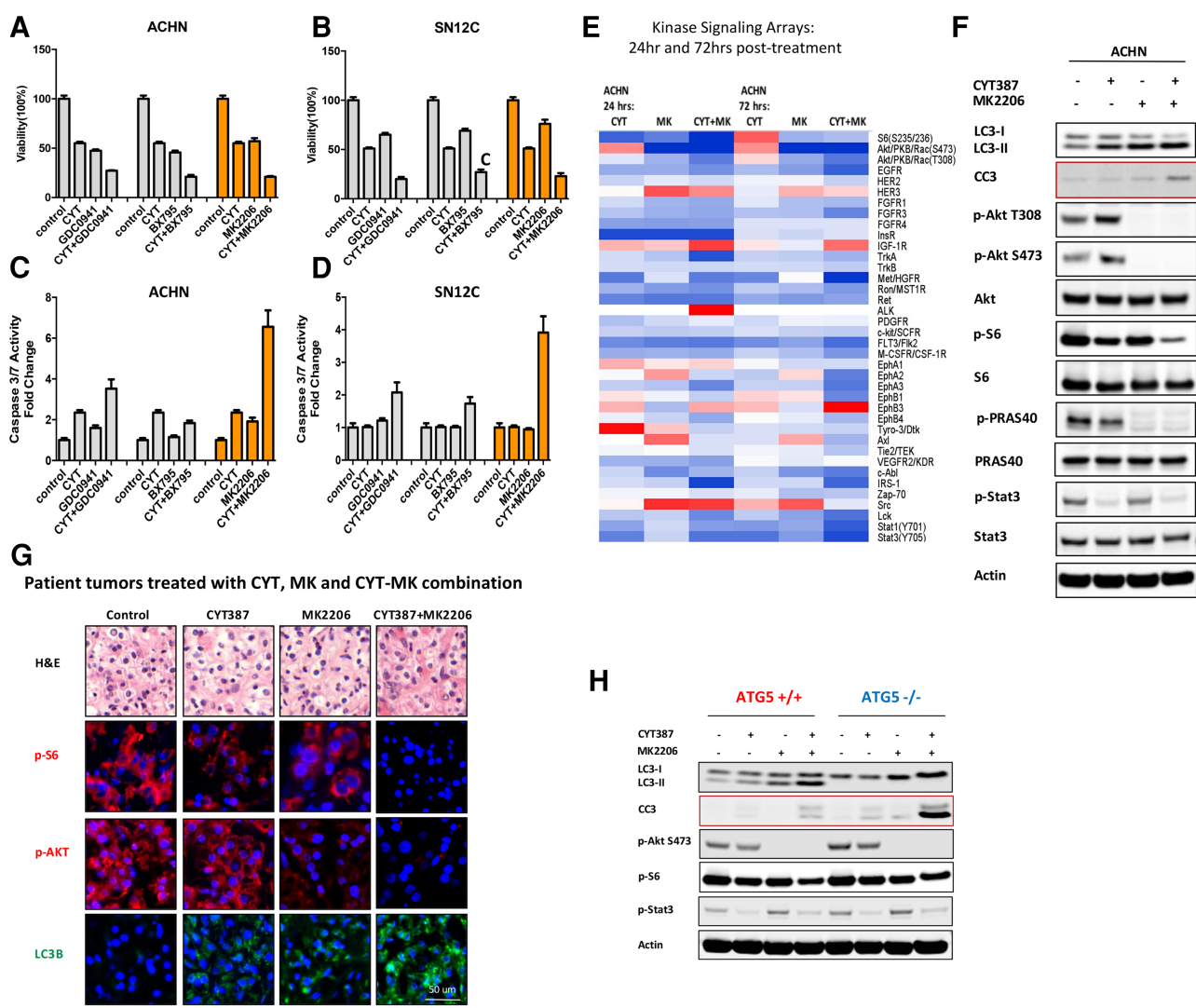

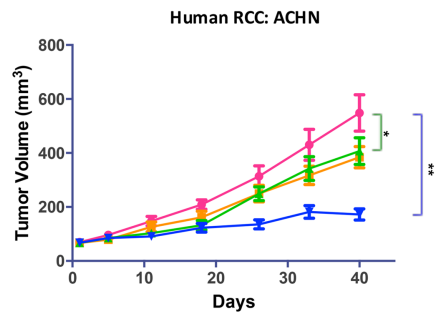

L

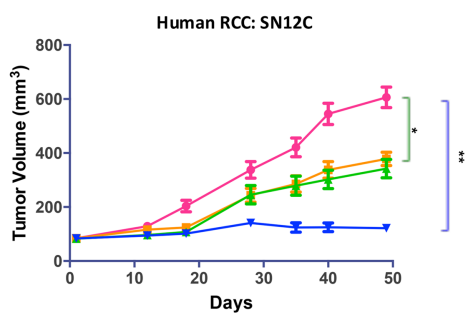

J

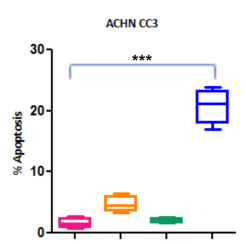

M

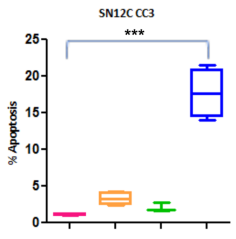

H

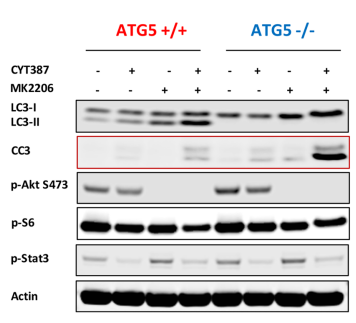

K

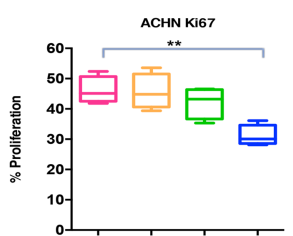

N

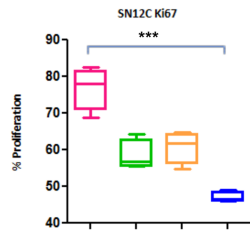

口 Control

군

口 ${ }^{\mathrm{MK}}$

- control

ㄷ мк

믐 $\mathrm{CrT}$ MKTT

Figure 2. CYT387 combines with MK2206 to effectively inhibit PI3K-AKT-mTOR signaling in human cancer cell lines and xenografts but does not induce tumor regression. $(A, B)$ Combination treatment with GDC0941, BX795, and MK2206 with measurement of cell viability in two human RCC cell lines: ACHN $(A)$ and SN12C $(B) .(C, D)$ The same combinations as in $A$, with measurement of apoptosis by cleaved caspase $3 / 7$ in $\mathrm{ACHN}(C)$ and SN12C $(D)$ cells. $(E)$ A heat map of signaling kinase arrays shows the effects of CYT387, MK2206, and CYT387+MK2206 cotreatment in ACHN cells at $24 \mathrm{~h}$ and $72 \mathrm{~h}$ after treatment. (F) Immunoblot for LC3, p-AKT Thr308, p-AKT Ser473, total AKT, p-PRAS40, total PRAS40, p-S6, total S6, p-STAT3, total STAT3, and $\beta$-actin. $(G)$ Patient-derived organotypic cultures treated with DMSO (control), CYT387, MK2206, and the CYT387+MK2206 combination for $24 \mathrm{~h}$ exhibit an increase in LC3B (green) and a decrease in p-S6 (red) and p-AKT (red). (H) ATG5 ${ }^{+/+}$and $A T G 5^{-/-}$MEFs were treated with $2 \mu \mathrm{M}$ CYT387, $10 \mu \mathrm{M}$ MK2206, and the combination for $24 \mathrm{~h}$, and LC3, cleaved caspase 3, p-AKT, p-S6, p-STAT3, and $\beta$-actin were evaluated by immunoblotting. (I) ACHN xenografts treated with vehicle, $50 \mathrm{mg} / \mathrm{kg}$ CYT387, $60 \mathrm{mg} / \mathrm{kg}$ MK2206, and a $50 \mathrm{mg} / \mathrm{kg}$ CYT387 + $60 \mathrm{mg} / \mathrm{kg}$ MK2206 combination. Tumor volume is shown. Error bars represent mean \pm SEM. Control versus CYT387+MK2206, $\left.{ }^{* *}\right) P<0.01$. $(J, K)$ The effect on apoptosis $(C C 3)(J)$ and proliferation $(\mathrm{KI} 67)(K)$ in ACHN xenograft tumors. Error bars represent mean \pm SEM. $(J)$ Control versus CYT387+MK2206, $\left({ }^{* * *}\right) P<0.0001$. $(K)$ Control versus CYT387+MK2206, ( $\left.^{* *}\right) P=0.0018$. (L) SN12C xenografts treated with vehicle, $50 \mathrm{mg} / \mathrm{kg} \mathrm{CYT387,} \mathrm{50} \mathrm{mg/kg} \mathrm{MK2206,} \mathrm{and}$

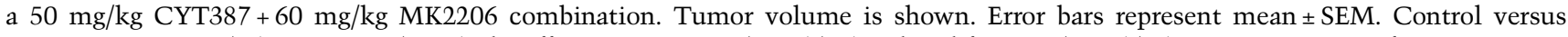
CYT387+MK2206, $\left(^{* *}\right) P<0.0001 .(M, N)$ The effect on apoptosis $(\mathrm{CC} 3)(M)$ and proliferation $(\mathrm{KI} 67)(N)$ in SN12C xenograft tumors. Error bars represent mean \pm SEM. $(M)$ Control versus CYT387+MK2206, $\left(^{* * *}\right) P<0.0001$. (N) Control versus CYT387+MK2206 $\left.{ }^{* * *}\right) P<0.0001$. 
mTOR pathway at proximal and distal nodes, CYT387 and MK2206 combine to shut down PI3K oncogenic signaling. However, autophagy still persisted in the combination treatment, pointing to a survival signal that sustains subpopulations of drug-tolerant cancer cells. Notably, the CYT387-MK2206 combination induced autophagy in patient-derived organotypic RCC cultures (Fig. 2G).

To further define the role of treatment-induced autophagy in mediating survival, we assessed the effects of CYT387 and MK2206 combination treatment on $A T G 5^{-/-}$and $A T G 5^{+/+}$MEFs. The CYT387-MK2206 cotreatment induced more apoptosis in ATG5 $5^{-1-}$ MEFS than it did in wild-type controls (demonstrated by an increase in cleaved caspase 3), indicating that autophagy protects cells from apoptosis (Fig. $2 \mathrm{H}$ ). Collectively, these results suggest that despite effective inhibition of PI3KAKT-mTOR signaling with the resultant induction of apoptosis, cancer cells are able to simultaneously induce an autophagic-fueled survival pathway.

We next examined the safety and efficacy of CYT387 and MK2206 cotreatment in vivo in two xenograft tumor models. While CYT387 or MK2206 alone exhibited an anti-tumor effect on ACHN and SN12C xenografts, the combination of CYT387 with MK2206 resulted in significantly greater tumor growth inhibition in ACHN and SN12C tumor xenografts $(P<0.001)$ (Fig. 2I,L). Importantly, combination treatment was well tolerated, with no weight loss recorded (Supplemental Fig. S3G,H). Pharmacodynamic studies demonstrated that combination therapy led to the suppression of S6 and AKTS473 phosphorylation (Supplemental Fig. S3I). Consistent with our in vitro finding, CYT387 alone had a minimal impact on apoptosis. In marked contrast, combination treatment with CYT387 and MK2206 resulted in a significant increase in apoptosis (established by an increase in cleaved caspase $3 ; P<0.001$ ) (Fig. 2J [ACHN xenograft tumors], $\mathrm{M}$ [SN12C xenograft tumors]) and a reduction in proliferation (demonstrated by a decrease in Ki-67; $P<0.001$ ) (Fig. $2 \mathrm{~K}$ [ACHN xenograft tumors], N [SN12C xenograft tumors]). However, despite effective inhibition of PI3KAKT-mTOR signaling, the combination treatment did not induce tumor regression.

\section{Metabolic reprogramming is supported by redox homeostasis}

The lack of tumor regression despite effective inhibition of PI3K-AKT-mTOR signaling led us to question whether metabolic reprogramming may sustain the survival of the treated cancer cells. The PI3K-AKT-mTOR pathway regulates multiple steps in glucose uptake and metabolism (Duvel et al. 2010). Therefore, we hypothesized that CYT387 and MK2206 treatment singly and in combination would negatively impact glucose uptake, aerobic glycolysis, and, subsequently, biosynthetic pathways, resulting in a drug-enforced reduction in glucose availability in the microenvironment. To determine the contribution of CYT387 and MK2206 treatment on the regulation of glycolysis, we measured glucose uptake by ${ }^{18}$ F-fluoro- deoxyglucose $\left({ }^{18} \mathrm{FDG}\right)$, lactate excretion, and the extracellular acidification rate (ECAR) as readouts for glycolysis. CYT387, MK2206, and the combination significantly decreased glucose uptake and reduced lactate production in vitro (Fig. 3A,B). The dramatic difference between lactate/ glucose ratio in extracellular medium further supports the finding that CYT387 and MK2206 cotreatment inhibits glycolysis (control: 1.51; CYT387: 0.65; MK2206: 0.81; CYT387+MK2206: 0.37). This impaired carbon metabolism with treatment also resulted in a reduction of cell size (Fig. 3C). Consistent with the above finding, CYT387, MK2206, and the CYT387-MK2206 combination significantly reduced the ECAR (Fig. 3D,E).

Decreased glucose availability with cotreatment might also be reflected in changes with OXPHOS activity, as measured by oxygen consumption rate $/ \mathrm{OCR}$; an indicator of OXPHOS). However, we found that the OCR/ECAR ratio increased after cotreatment, suggesting a predominant decrease in glycolysis with the maintenance of mitochondria-driven OXPHOS (Fig. 3F). Consistent with glucose limitation and decreased glycolysis, we observed increased AMPK phosphorylation at Thr172, an established indicator of metabolic stress (Fig. 3G). Importantly, in the setting of glucose deprivation and impairment of the pentose phosphate pathway (PPP), AMPK has been shown to increase NADPH levels from increased fatty acid oxidation. Specifically, we noted increased levels of NADPH, maintenance of GSSG/GSH ratios, and a resultant mitigation of reactive oxygen species (ROS) (Fig. 3H-J). These findings are consistent with the role of AMPK in mitigating metabolic stress and promoting cancer cell survival (Jeon et al. 2012). Additionally, AMPK would be predicted to further inhibit mTOR (Inoki et al. 2003; Gwinn et al. 2008). By comparison, we did not see any reduction in PKM2 levels, suggesting that the metabolic switch from aerobic glycolysis to OXPHOS is not dependent on pyruvate kinase activity (Christofk et al. 2008).

Overall, these findings suggest that by decreasing glucose levels, CYT387-MK2206 cotreatment severely reduces the glycolytic capacity needed to supply the bioenergetics needs of the RCC cells. Importantly, this treatment-induced nutrient-depleted condition, while suppressing proliferation, simultaneously promotes survival by regulating NADPH homeostasis and maintaining mitochondrial-driven oxidation.

\section{PI3K-AKT-mTOR treatment-induced autophagy promotes phospholipid metabolism}

Therefore, to comprehensively determine how autophagy contributes to the metabolic needs, we performed global metabolic analysis using a liquid chromatography-tandem mass spectrometry (LC-MS/MS)-based platform (Louie et al. 2016). These studies revealed that CYT387 and MK2206, singly and in combination, effected changes across multiple pathways (Fig. 4A; Supplemental Table 14). Consistent with the role of the PI3K-AKT-mTOR pathway in the regulation of glycolysis, treatment with these agents was accompanied by reductions in glucose, glucose-6-phosphate, DG3P, PEP, pyruvate, and lactate, 


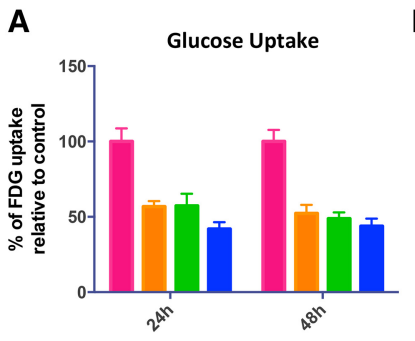

D

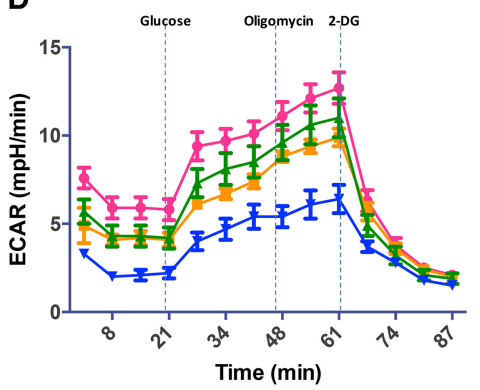

$a^{2}$

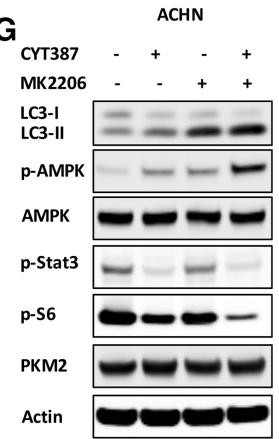

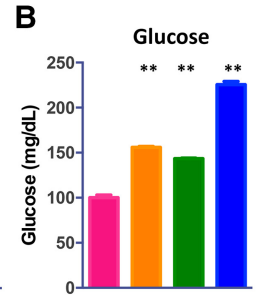

E

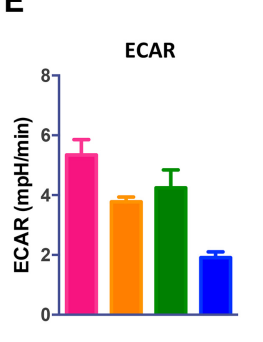

I

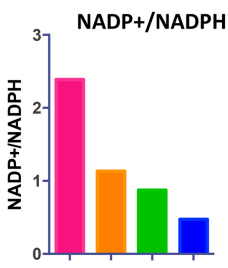

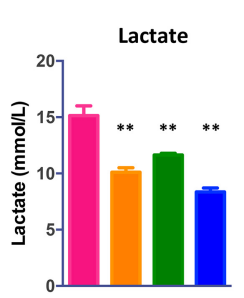

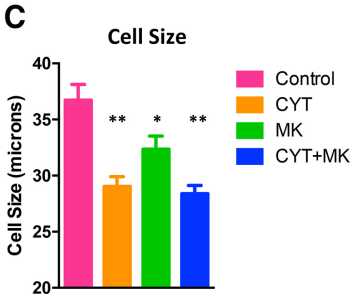

$\mathbf{F}$
OCR/ECAR

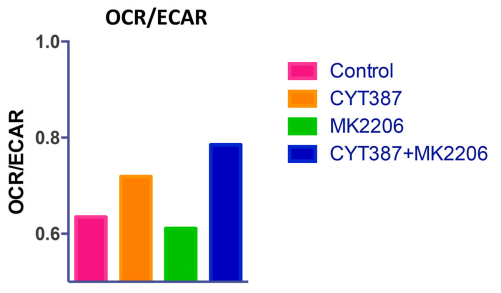

J

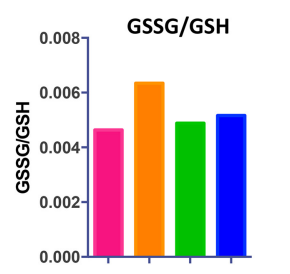

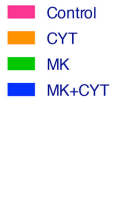

Figure 3. The effects of treatment on metabolism. (A) The treatment effects of control, CYT387, MK2206, and CYT387+MK2206 on glucose uptake over time, measured by ${ }^{18} \mathrm{FDG}$. $(B)$ Glucose and lactate levels in culture medium were measured in control and treated cells and normalized to cell number. (C) Qualitative analysis of cell diameter changes of ACHN cells treated with CYT387, MK2206, CYT387 + MK2206, or vehicle (DMSO). $\left.{ }^{*}\right) P<0.02$. (D) Glycolysis in ACHN cells was measured using a XF-96 extracellular flux analyzer after preincubation with drugs or DMSO. Shown are ECAR means \pm SD of experimental triplicates. $(E)$ The effects of treatment on basal ECAR, measured in real time and presented as change in milli-pH per unit time. Representative results are shown. $n=2$. $(F)$ Ratios of oxygen consumption rate (OCR; an indicator of OXPHSO) to ECAR (an indicator of aerobic glycolysis at baseline) of treated ACHN cells. Representative results are shown. $n=2$. $(G-J)$ Treatment activates p-AMPK and increases NADPH levels, maintains GSSG/GSH ratios, and mitigates reactive oxygen species (ROS). (G) ACHN cells were treated with control, $2 \mu$ M CYT387, $10 \mu M$ MK2206, and CYT387 + MK2206 for $24 \mathrm{~h}$ and probed with the indicated antibodies. (H) NADP $/ \mathrm{NADPH}$ levels were measured. (I) GSSG and GSH levels were measured in lysates $(n=4)$ using liquid chromatography-tandem mass spectrometry (LC-MS/MS). ( $J$ ) The normalized abundance of these metabolites is shown. Cells were stained with the ROS sensor CellRox. $n=3$; three independent experiments. $P=$ ns.

consistent with the inhibition of glycolysis (Supplemental Fig. S4A), as described above and also concordant with the gene expression data. Similarly, we also observed reductions in PPP intermediates, amino acids, tricarboxylic acid (TCA) cycle intermediates, and ribose biosynthesis and corresponding increases in purine breakdown products guanine and hypoxanthine (Supplemental Fig. S4BE). These findings are in keeping with a nutrient-deprived state (i.e., decreased anabolism) with subsequent increased autophagic catabolism to maintain survival (Mizushima et al. 2001). Cells adapt to glucose deprivation by subsisting on fatty acids-mobilized through glycerolipid remodeling-for oxidation, and this is consistent with our observation that the most significant metabolite changes were in lipid intermediates, including phospholipids, triacylglycerol (TAG), cholesterol esters, diacylglycerol (DAG), and fatty acids (C16:0, C18:0, and C18:1) (Fig. 4A; Supplemental Fig. S4F; Kerner and Hoppel 2000; Eaton 2002; Finn and Dice 2006).

We further investigated the lipid substrates that were catabolized by autophagy to produce fatty acids for fatty acid oxidation. Steady-state metabolite profiling showed significant increases in lysophospholipids and arachidonic acid (C20:4), with corresponding decreases in their phospholipid precursors (Fig. 4B). Phospholipids, which include phosphatidylcholine $(\mathrm{PC})$, phosphatidylethanolamine (PE), 
A

Metabolic Profiling
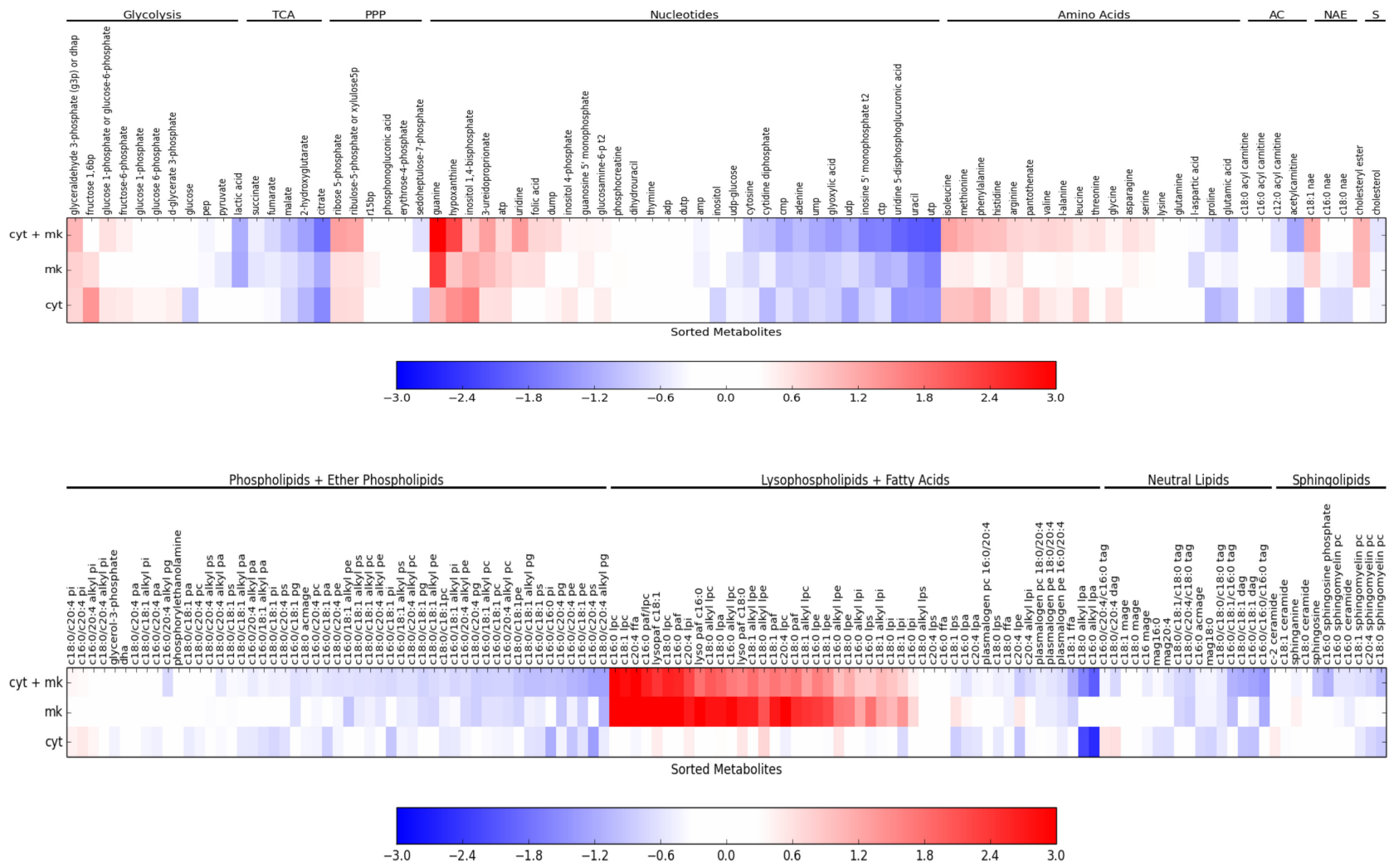

B

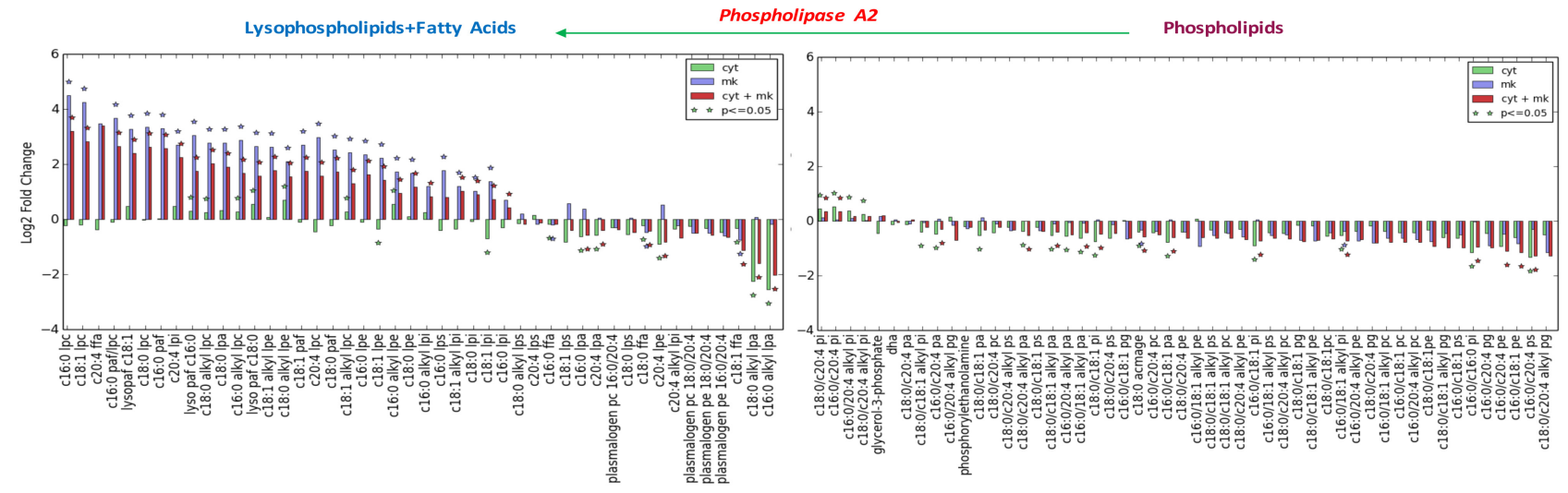

Figure 4. PI3K-AKT-mTOR signaling inhibition induces metabolic reprogramming. (A) Heat map of metabolomic profiling of treated cells (CYT387, MKK2206, and CYT387+MMK2206) compared with ACHN cells treated with vehicle (DMSO) using LC-MS/MS (see the Materials and Methods for details). Log fold changes of profiled metabolites are shown. Metabolites are ordered within each category: glycolysis, TCA cycle, PPP, nucleotide metabolism, amino acid metabolism, phospholipids, ether phospholipids, lysophospholipids, fatty acids, neutral lipids, acyl carnithines (AC), sphingolipids (SL), and n-acyl ethanolamines (NAE). (B) PLA2 catalyzes the hydrolysis of phospholipids to lysophospholipids and arachidonic acid. Waterfall plot demonstrating relative levels of phospholipids, lysophospholipids, and fatty acids in treated cells (CYT387, MK2206, and CYT387+MK2206) compared with ACHN cells treated with vehicle (DMSO). Asterisks denote a significant difference of treated cells compared with vehicle $(t$-test, $P$-value $\leq 0.05)$.

phosphatidylserine (PS), phosphatidylglycerol (PG), and phosphatidylinositol (PI), are major structural components of cellular membranes. PLA2 is the enzyme that catalyzes the hydrolysis of the phospholipid sn-2 ester bond with subsequent release of lysophospholipids; e.g., lysophosphatidylcholine (LPC), alkly-lysophosphatidylcho- line (alkyl-LPC), and free fatty acids (Murakami et al. 2011). Accordingly, we found elevated levels of C16:0 LPC, C18:0 LPC, C18:1 LPC, and C18-0 alkyl-LPC and corresponding decreases in their phospholipid precursors. Notably, we observed significant decreases in free fatty acids (C16:0, C18:0, and C18:1), supporting the idea that 
phospholipids are hydrolyzed to supply fatty acids for fatty acid oxidation. Consistent with increased arachidonic acid levels in CYT387-MK2206-cotreated cells, we observed increased levels of 14,15-EET, 11,12-EET, 8,9-EET, and 5HETE, pointing to arachidonic acid $\mathrm{P} 450$-mediated generation of eicosanoids (Supplemental Fig. S5).

\section{PI3K-AKT-mTOR treatment-induced autophagy facilitates lipid droplet (LD) formation and mitochondrial respiration}

To protect cells from the destabilizing effects of excess lipids, free fatty acids mobilized by autophagy and destined for oxidation are stored in an intermediate intracellular pool: LDs (Thiam et al. 2013). We reasoned that the large changes in glycerolipid redistribution identified by our metabolomics profiling of treated cells would result in an increased number of LDs to support fatty acid oxidation, with subsequent mobilization of fatty acids to mitochondria under these nutrient-depleted conditions (Rambold et al. 2015). Consistent with this, we observed that CYT387 and MK2206 singly and in combination incrementally and significantly increased the number and size of Bodipy 493/503-labeled (Fig. 5A-C, green) LDs. Additionally, we incubated ACHN human RCC cells with Bodipy-C12-HPC (a phospholipid containing green fluorescent long chain fatty acid) followed by treatment with vehicle or the CYT387-MK2206 combination. CYT387-MK2206 cotreatment led to a greater degree of incorporation of Bodipy-C12-labeled fatty acids into LDs relative to vehicle-treated cells. This suggests that CYT387-MK2206 treatment-induced autophagy results in phospholipid hydrolysis that releases fatty acids, which are subsequently incorporated into new LDs (Supplemental Fig. S6).

To determine whether the increase in LDs occurred in vivo, we stained the vehicle, CYT387, MK2206, and CYT387-MK2206-cotreated xenograft tumors for adipophilin, which belongs to the perilipin family, members of which coat intracellular lipid storage droplets and facilitate metabolic interactions with mitochondria (Sztalryd and Kimmel 2014). Consistent with the in vitro data, the number of adipophilin-positive LDs significantly and incrementally increased with treatment (as measured on treatment day 40 in ACHN xenograft tumors; CYT387<MK2206<CYT387+MK2206; $P=0.0046$ ) (Fig. $5 \mathrm{D})$, indicating that these drug treatments stimulate the formation of LDs in vivo. Collectively, these data suggest that the early adaptive and survival changes effected by the initial drug treatment continues to support the maintenance of long-term in vivo tumor growth.

Next, to further determine whether autophagy contributed to LD numbers, we treated $A T G 5^{+/+}$and $A T G 5^{-/-}$ MEFs with CYT387, MK2206, and the combination. Autophagy-competent ATG5 ${ }^{+/+}$MEFs were able to significantly increase LD numbers (Fig. 5E). In marked contrast, none of the treatments was able to increase LDs in $A T G 5^{-/-}$MEFs, confirming that autophagy is required to sustain LD levels (Fig. 5F). To investigate the metabolic ramifications of this, we compared oxygen consumption by $A T G 5^{-/-}$and $A T G 5^{+/+}$MEFs when treated with CYT387, MK2206, and the combination. We found that CYT387-MK2206 cotreatment profoundly decreased the

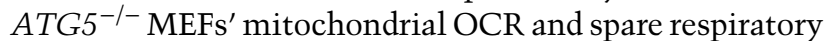
capacity (SRC; the quantitative difference between the maximal OCR and the initial basal OCR), indicating that $A T G 5^{-1-}$ MEFs function near their maximal rate and are unable to maintain an adequate level of mitochondrial respiration with CYT387+MK2206 cotreatment due to a deficit in their metabolic compensatory mechanisms (Fig. 5G,H). In contrast, we observed no decrease in mitochondrial OCR and SRC in CYT387-MK2206-cotreated $A T G 5^{+/+}$MEFs.

The extent of the metabolic difference between vehicletreated $A T G 5^{-/-}$and CYT387+MK2206-cotreated MEFs was apparent in the overall ratio of OXPHOS to aerobic glycolysis (OCR/ECAR), which was twofold higher in vehicle-treated than in CYT387-MK2206-cotreated $A T G 5^{-/-}$MEFs, reflective of the need for autophagy to supply the nutrients to maintain mitochondrial OCR under treatment-enforced metabolic restrictions (Fig. 5I).

This is in line with a model in which autophagy of cellular organelles and membranes during nutrient deprivation produces fatty acids that supply the LD pool, where they are then transferred into mitochondria for $\beta$-oxidation. In support of this, we observed that treated RCC cells had significantly increased numbers of mitochondria (Fig. 5J). Accordingly, dual staining of treated ACHN cells with a mitochondrial marker (Mitotracker orange) and LDs with Bodipy (Fig. 5K, green) revealed that the LDs were closely associated with the mitochondria, potentially enabling the fatty acids released from LDs to traffic directly from LDs to mitochondria and maximizing the fatty acid oxidation (Fig. 5K; Rambold et al. 2015).

Importantly, cancer cells become increasingly dependent on mitochondrial fatty acid oxidation in nutrient-depleted conditions (Fig. 5L; Cabodevilla et al. 2013). Consistent with this, using the Mito Fuel Flex test, we found that human ACHN RCC cells' dependence on fatty acid doubled with CYT387-MK2206 cotreatment (Fig. $5 \mathrm{M})$. Consequently, oxidation of endogenous fatty acids significantly contributed to the OXPHOS rate in MK2206+CYT387-cotreated cells compared with control (>2.5-fold increase; $P<0.0001$ ) (Fig. $5 N$ ). Consistent with this, induction of fatty acid oxidation by CYT387MK2206 cotreatment was attenuated in $A T G^{-/-}$MEFs (Supplemental Fig. S7). In contrast, glutamine-supported OCR represented a minority of total OCR in CYT387MK2206-cotreated ACHN cells (Supplemental Fig. S8). Taken together, this suggested that cellular lipid remodeling by the autophagy-lysosome system may supply a considerable fraction of the intracellular lipids-fatty acids irrespective of their external availability.

Inhibiting PLA2 activity decreases autophagy-induced LDs, limits OXPHOS, and increases apoptosis

Our data implicated hydrolysis of phospholipids as a critical mechanism for the generation of lysophospholipids 


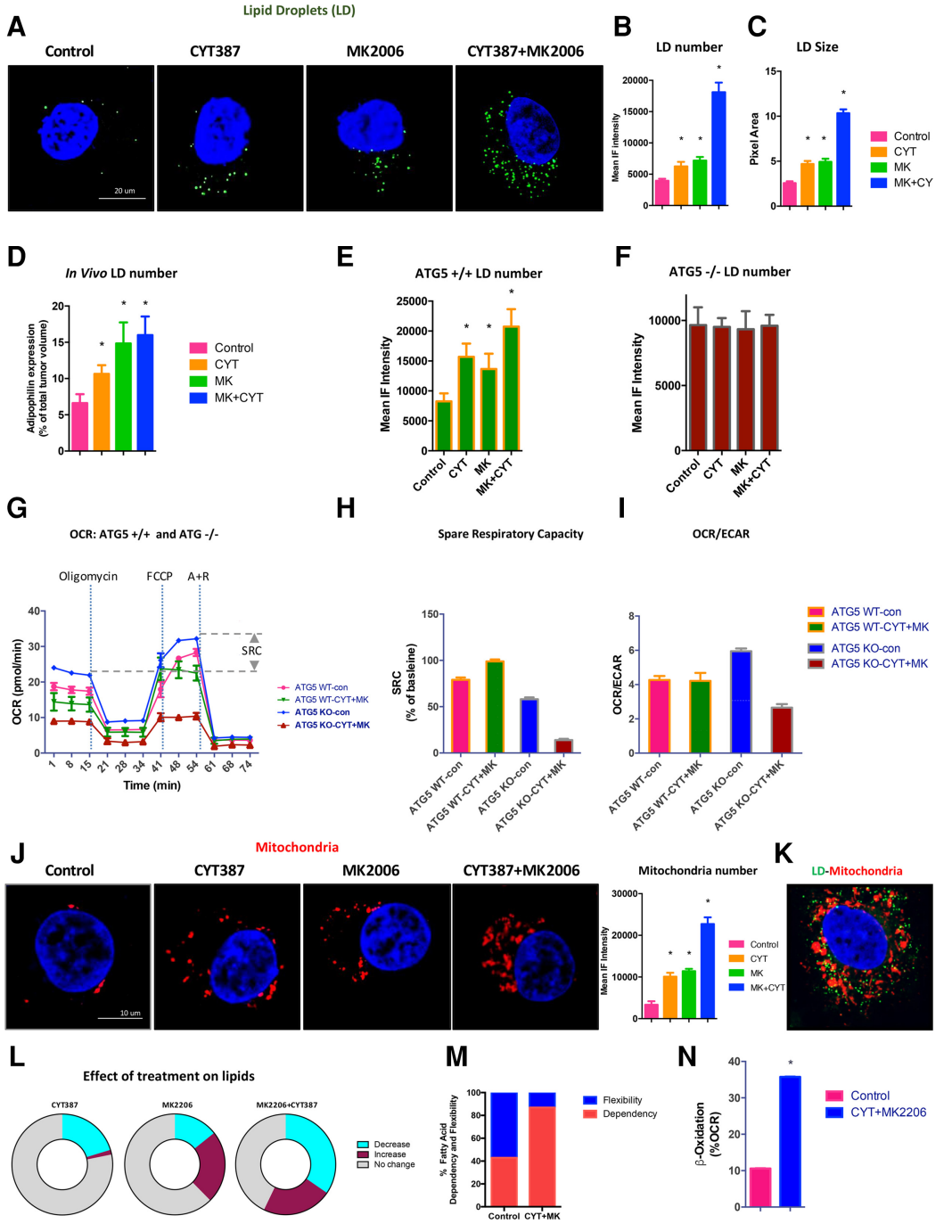

Figure 5. Autophagy is required for LD growth and fatty acid oxidation. (A) ACHN cells were treated with control, CYT387, MK2206, and CYT387+MK2206 for 24 h, and Bodipy 493/503 (green) was added to visualize LDs. Representative images are shown. $n=5$ experiments. $(B, C)$ Bar graphs quantify the increase in number $(B)$ and size $(C)$ of LDs, respectively. Data are expressed as means \pm SEM. $\left(^{*}\right) P<0.001$ for control versus CYT387, control versus MK2206, and control versus CYT387+MK2206. (D) Adipophilin staining in xenograft tumors quantifies the increase in LDs in vivo. $n=9$. Data are expressed as means \pm SEM. $\left({ }^{*}\right)$ $P<0.01$ for control versus CYT387 and control versus MK2206, measured in tumors resected after $40 \mathrm{~d}$ of treatment. (E) $A T G 5^{+/+}$MEFs were treated with $2 \mu \mathrm{M}$ CYT387, $10 \mu \mathrm{M}$ MK2206, and the combination for $24 \mathrm{~h}$. Bodipy was added, and the LD number was measured. $n=500$ cells. $\left.{ }^{*}\right) P$ $<0.001$ for control versus CYT387 and control versus CYT387+MK2206; $P<0.005$ for control versus MK2206. (F) ATG5 ${ }^{-1-}$ MEFs treated as in E. Bodipy was added, and the LD number was measured. $n=500$ cells. $P=$ NS (no significance between treatment groups). (G) $A T G 5^{+/+}$and $A T G 5^{-/-}$ MEFs were treated with DMSO (control), CYT387, MK2206, and CYT387+MK2206 for 24 $h$, and then OCRs (indicator of OXPHOS) were determined using a XF-96 extracellular flux analyzer during sequential treatments (dotted vertical lines) with oligomycin, FCCP, and rotenone/ anti-mycin $(\mathrm{A}+\mathrm{R})$. Spare respiratory capacity (SCR) is the quantitative difference between maximal uncontrolled OCR (top horizontal dashed line) and the initial basal OCR (bottom horizontal dashed line). Shown are OCR means \pm SD of experimental triplicates. For ease of viewing, only control and CYT387 + MK2206 data are graphed. $(H)$ SRC (the percentage maximum OCR after FCCP injection of baseline OCR) of $A T G 5^{+/+}$and $A T G 5^{-/-}$MEFs after the indicated treatments. Shown are means \pm SD of experimental triplicates. (I) Ratios of OCR to ECAR (indicator of aerobic glycolysis) at baseline of $A T G 5^{+/+}$ and $A T G 5^{-1-}$ MEFs after the indicated treatments. (J) ACHN cells were treated with control, CYT387, MK2206, and YT387+MK2206 for $24 \mathrm{~h}$, and Mitotracker orange was added to visualize mitochondria. Representative images are shown. $n=5$ experiments. Mitochondria number was measured, and data are expressed as means \pm SEM. $\left(^{*}\right) P<0.001$ for control versus CYT387, control versus MK2206, and control versus MK2206+CYT387. (K) Dual staining of Bodipy and Mitotracker orange demonstrate close proximity of LDs with mitochondria in CYT387+MK2206-cotreated ACHN cells (a representative image is shown). $(L)$ Global metabolite profiling reveals a preferential decrease in lipids. (Decrease) Abundance $<0.5$-fold in treated cells compared with the vehicle; (increase) abundance greater than twofold in treated cells compared with the vehicle. $(M)$ Fatty acid fuel dependency measures the reliance of ACHN cells on fatty acids to maintain baseline respiration. ACHN cells were treated with DMSO (control) or CYT387+MK2206 for $24 \mathrm{~h}$, and OCR was measured during the Seahorse XF Mito Fuel Flex assay. The percentage of dependence on fatty acids was calculated by quantifying the change in basal OCR after fatty acid oxidation was blocked using $4 \mu \mathrm{M}$ CPT-1a inhibitor etomoxir divided by the total change in OCR from baseline after combined inhibition of fatty acid, glutamine, and pyruvate oxidation using $4 \mu \mathrm{M}$ eomoxir, $3 \mu \mathrm{M}$ BPTES, and $2 \mu \mathrm{M}$ UK5099, respectively (representative graph). $n=2$. Fatty acid fuel flexibility was calculated by measuring the change in sensitivity to etomoxir's inhibition of OCR after blockade of glutamine and glucose oxidation and represents the ability of ACHN cells to increase oxidation of fatty acid when glutamine and pyruvate utilization is precluded. $(N)$ Measurement of fatty acid-driven OCR, measured by acute inhibition of CPT-1a using 4 $\mu \mathrm{M}$ etomoxir $\left(\left[{ }^{*}\right] P<0.01\right)$ and represented as a percentage of total mitochondria OCR calculated using mitochondrial complex I and III inhibitors $1 \mu \mathrm{M}$ rotenone and $1 \mu \mathrm{M}$ anti-mycin, respectively.

and fatty acids for fatty acid oxidation in treated RCC cells, and, therefore, inhibition of this enzymatic activity would negatively impact OXPHOS and subsequently limit the survival of these cells. To test this directly, we added the PLA2 inhibitor oleyloxyethylphosphocholine
(OOEPC; which inhibits secretory PLA) (Slatter et al. 2016) to CYT387, MK2206, and CYT387-MK2206cotreated cells and measured LD numbers. Addition of OOEPC significantly reduced the LD abundance in CYT387, MK2206, and CYT387-MK2206-cotreated cells 
(Fig. 6A,B). Since several isoforms of PLA2 exist, we determined their role in reducing LDs. We found that inhibition of calcium-sensitive PLA2 (with cPLA2i) and calcium-insensitive PLA2 (with bromoenol lactone [BEL]) was also able to reduce LD number, consistent with the rate-limiting role of PLA2 in mediating phospholipid hydrolysis (Supplemental Fig. S9). To document the kinetics of the new pool of CYT387-MK2206-induced LDs, we performed a time-course experiment to monitor the appearance of LDs following CYT387-MK2206 cotreatment and ascertained that LDs appeared $2 \mathrm{~h}$ after treatment and then continuously increased in number during the next $24 \mathrm{~h}$ of monitoring. In contrast, simultaneous addition of OOPEC to the CYT387-MK2206 combination at the start of treatment completely blocked the appearance of LDs. Similarly, addition of OOPEC at $2 \mathrm{~h}$ after cotreatment with CYT387+MK2206 completely inhibited any further increase in LDs. Subsequently, the addition of etomoxir at $8 \mathrm{~h}$ (which blocks the utilization of fatty acids) resulted in LD accumulation in OOPEC+CYT387+MK2206-treated cells. These results demonstrate that PLA2 activity is required for LD generation after CYT387-MK2206 cotreatment and that OOPEC is able to inhibit PLA2 activity (Supplemental Fig. S10).

To directly test the metabolic impact of OOEPC treatment, we first assessed changes in the OCR. We observed a marked decrease in the basal OCR when OOEPC was added to the CYT387-MK2206 combination. Importantly, the addition of OOPEC profoundly reduced the SRC, indicating that the inhibition of PLA2 decreases mitochondrial oxidation by reducing fatty acid supply and impedes the cells' capacity to respond to increased energetic demands (Fig. 6C,D). The marked reduction in SRC was similar to our earlier observations in CYT387-MK2206treated $A T G 5^{-/-}$MEFs and is consistent with the model in which autophagy-supplied LDs are required to support mitochondrial OCR in metabolically restricted environments (Fig. 5G-I). Next, by plotting OCR versus ECAR, we determined the effect of PLA2 inhibition by OOEPC on CYT387-MK2206-treated tumors; this measurement highlighted that untreated ACHN human RCC cells have higher OXPHOS and glycolysis compared with CYT387-MK2206-cotreated cells (Fig. 6E). The addition of OOEPC markedly decreased OCR in ACHN cells, indicating that these treatments diminished the overall metabolic activity of the cancer cells.

This observed reduction in bioenergetic metabolism led us to determine whether PLA2 inhibition would have an impact on proliferation and apoptosis. Cotreatment with OOEPC had a minimal additional effect on proliferation (Fig. 6F). In contrast, the addition of OOEPC significantly increased apoptosis, consistent with its ability to reverse autophagy-supplied fatty acids that enable survival (Fig. 6G). To further verify that PLA2 inhibition impacted cancer cell survival, we tested a distinct PLA2 inhibitor, varespladib, which has been clinically developed for cardiovascular diseases (Rosenson et al. 2010). Similar to OOEPC, the addition of varespladib to CYT387MK2206-treated cells decreased LDs and increased apo- ptosis (Fig. 6H-J). Collectively, these data indicate that treatment-induced autophagy provides lysophospholipids and free fatty acids to maintain cancer cell survival despite nutrient depletion.

\section{Discussion}

It is now generally accepted that autophagy is cytoprotective in the setting of cancer therapies by enabling cancer cells to mitigate metabolic and therapeutic stresses, thereby ensuring survival (Amaravadi et al. 2011; Sehgal et al. 2015; Rebecca and Amaravadi 2016). To date, the therapeutic reflex to block autophagy is to add antimalarial lysosomotropic inhibitors such as chloroquine. However, the clinical responses to these have been underwhelming (Goldberg et al. 2012; Shanware et al. 2013; Rosenfeld et al. 2014; Towers and Thorburn 2016). While the role of autophagy in tumor initiation and progression has been well-documented, little is known about how treatment-induced autophagy mediates cytoprotection and resistance.

Our results demonstrate that cancer cells, when acutely exposed to small molecule inhibitors, activate the autophagic process to ensure early and lasting metabolic adaptations designed to enhance survival in a nutrientdepleted environment. We first observed the maintenance of OXPHOS when glucose became limiting due to treatment. Likewise, the coordinate activation of AMPK signaling ensures protective redox homeostasis to mitigate increased ROS produced by OXPHOS. Finally, we demonstrated activation of autophagy-mediated membrane glycerophospholipid metabolism with subsequent fatty acid oxidation to generate energy. Accordingly, we found that therapy-induced autophagy purposefully harnesses core biological processes to secure tumor cell fitness and survival. Our experiments involving autophagy-incompetent $A T G 5^{-/-}$MEFs demonstrate that autophagy is required under conditions of nutrient depletion to generate LDs and maintain mitochondrial OCR and SRC. It is not coincidental that LD depletion by pharmacological PLA2 inhibition achieved similar results. This is consistent with the model that autophagic digestion of phospholipids, with subsequent hydrolysis within the autolysosome, provides LDs with a constant supply of lipids, which can then be trafficked to the mitochondria to maintain mitochondrial respiration. The subsequent release of these fatty acids from LDs to fuel $\beta$-oxidation may occur independently of lipophagy, as others have observed (Rambold et al. 2015). Additionally, another possible source of fatty acids and amino acids may come from extracellular lysophospholipids and proteins through macropinocytosis.

This study further addresses the wider question of how cancer cells survive despite the inhibition of mTOR /an evolutionarily conserved master regulator of cell metabolism, proliferation, growth, and survival) and AKT (a committed prosurvival kinase that positively regulates these same processes in both normal and cancer cells) (Manning and Cantley 2007; Laplante and Sabatini 2012). 
A

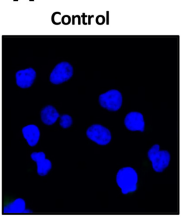

OOPEC
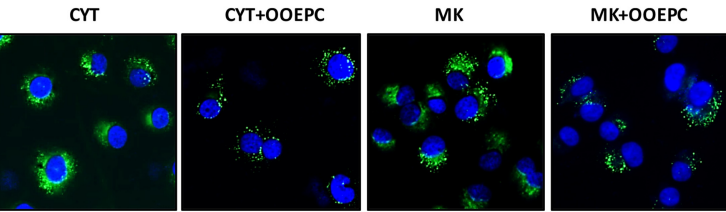

$\mathrm{MK}+\mathrm{CYT}$ MK+CYT+OOEPC
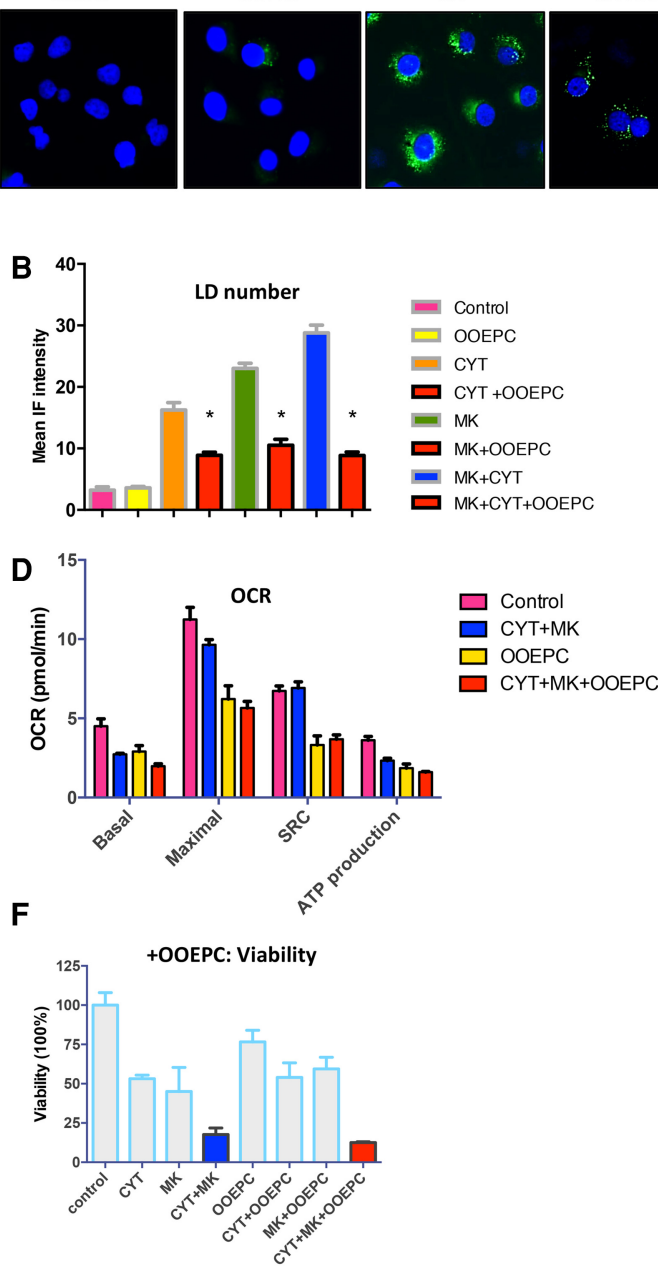

$\mathbf{H}$

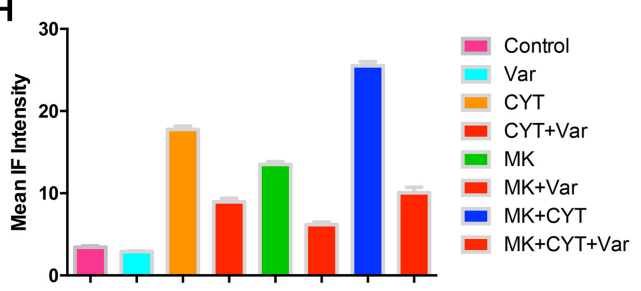

I

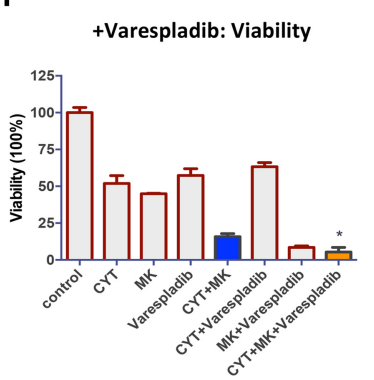

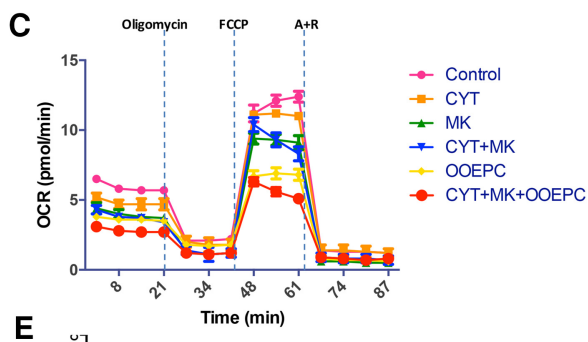

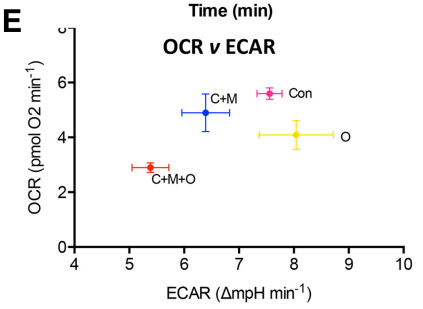

G
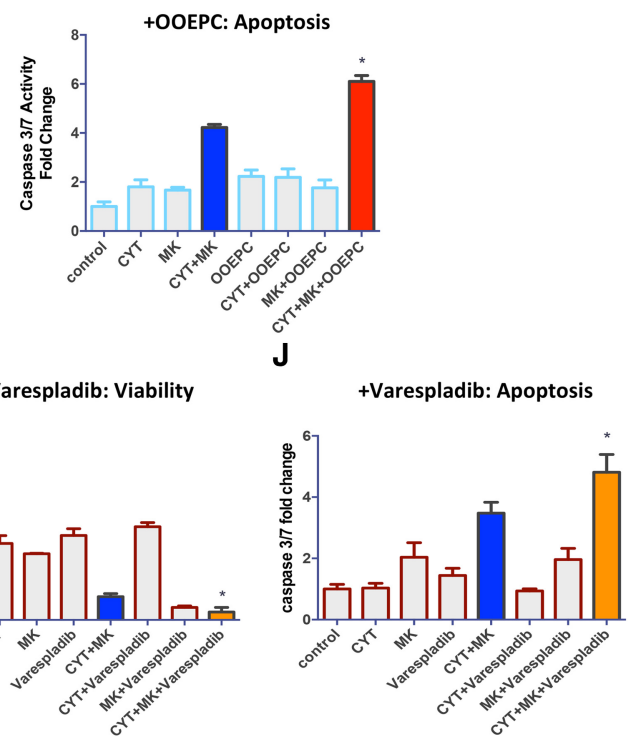

Figure 6. Hydrolysis of phospholipid supplies lysophospholipids and fatty acids for cancer cell survival. $(A)$ ACHN cells were treated with control, OOEPC, CYT387, CYT387+OOEPC, MK2206, MK2206+OOEPC, CYT387+MK2206, and CYT387+MK2206+OOEPC for 24 h. Bodipy 493/503 (green) was added to visualize LDs. Representative images are shown. $n=3$ experiments. (B) Bar graphs quantify the number of LDs. Data are expressed as means \pm SEM. $(*) P<0.0001$ for CYT387 versus CYT387+OOEPC, MK2206 versus MK2206 +OOEPC, and CYT387+MK2206 versus CYT387+MK2206+OOEPC. (C,D) ACHN cells were treated with DMSO (control), OOEPC, CYT387, CYT387+OOEPC, MK2206, MK2206+OOEPC, CYT387+MK2206, and CYT387+MK2206+OOEPC for $24 \mathrm{~h}$, and then OCR was determined using a XF-96 extracellular fux analyzer during sequential treatments with oligomycin, FCCP, and rotenone/anti-mycin $(\mathrm{A}+\mathrm{R})$. (D) Initial basal OCR, maximal OCR, SRC (the quantitative difference between maximal uncontrolled OCR and the initial basal OCR), and ATP production are depicted in the plot. Shown are OCR means \pm SD of experimental triplicates. For ease of viewing, only control, OOEPC, CYT387+MK2206, and CYT387+MK2206+OOEPC data are graphed. (E) OCR versus ECAR (means \pm SEM, experimental triplicates) after the addition of OOEPC to the CYT387-MK2206 combination. (Con) Control; (O) OOEPC; (C+M) CYT387+MK2206; $(\mathrm{C}+\mathrm{M}+\mathrm{O}) \mathrm{CYT} 387+\mathrm{MK} 2206+\mathrm{OOEPC}$. $(F, \mathrm{G})$ Cell viability $(F)$ and caspase $3 / 7$ activity $(G)$ with addition of OOEPC to CYT387, MK2206, and CYT387+MK2206. $n=3$. Data are expressed as means \pm SD. $(F) P=$ ns for CYT387+MK2206 versus CYT387+MK2206 +OOEPC. $(G)(*) P<0.001$ for CYT387+MK2206 versus CYT387+MK2206+OOEPC. $(H)$ The effect of adding varespladib, a distinct PLA2 inhibitor, to CYT387, MK2206, and CYT387+MK2206 on LD numbers was evaluated with Bodipy staining $(I, J)$ Cell viability $(I)$ and caspase $3 / 7$ activity $(J)$ with the addition of varespladib, a distinct PLA2 inhibitor, to CYT387, MK2206, and CYT387+MK2206. $n$ $=3$. Data are expressed as means \pm SD. $(I)(*) P<0.01$ for CYT387+MK2206 versus CYT387+MK2206+varespladib. $(J)(*) P<0.1$ for CYT387+MK2206 versus CYT387+MK2206+varespladib. 
Undoubtedly, the combination of attenuated proliferation signals, nutrient depletion, and metabolic competition for remaining nutrients kills many cells. Accordingly, our data demonstrate that glucose, which is tightly regulated by the PI3K-AKT-mTOR pathway at multiple steps, became limiting with treatment, with a resultant decrease in glycolysis (Engelman et al. 2006; Yecies and Manning 2011; Hu et al. 2016). However, the very same conditions that give rise to these nutrient-deprived microenvironments also induced autophagy. Consequently, the autophagic catabolism of membrane phospholipids provides a ready source of free fatty acids that maintains respiration in subpopulations of cancer cells, therefore enabling their survival in a low-glucose environment. The increase in fatty acid oxidation and OXPHOS requires redox homeostasis, and this is provided by the concomitant activation of AMPK, which increases NADPH, with a subsequent mitigation of ROS. Collectively, treatment-enforced metabolic reprogramming supports cancer cell fitness by providing fatty acids and NADPH to maximize survival.

Since the rate of autophagic release of fatty acids does not match the rate of mitochondrial consumption, these LDs serve a dual purpose: first, as a buffer to reduce lipotoxicity by storing lipid intermediates and, second, to transport these lipids to the mitochondria (Singh et al. 2009; Unger et al. 2010; Rambold et al. 2015). Consequently, these energy-strapped residual cancer cells increase fatty acid oxidation, as it is the most energetically efficient way to generate ATP. Long-lived cell types such as cardiac myocytes and memory $\mathrm{T}$ cells (Pearce et al. 2009; Chung et al. 2010) depend on fatty acid metabolism for survival, and we see this as yet another example of cancer cells hijacking normal physiological processes to their benefit.

Our screen identified several structurally different Janus family kinase inhibitors that inhibited mTORC1 and induced autophagic flux. While serendipitous, these findings are not unexpected, as small molecules inhibit several kinases and would directly and/or indirectly interdict the PI3K-AKT-mTOR pathway. To date, JAK inhibitors have been approved for and/or are undergoing late stage clinical trials in MPN, including the focus of this study, CYT387 (momelutinib) (Patel et al. 2016; Winton and Kota 2017). However, complete cytogenetic or molecular responses with JAK inhibitors have not been observed, with clinical benefit mainly resulting from improved performance status due to reduced cytokine levels rather than the elimination of cancer cells /Verstovsek et al. 2012; Vannucchi et al. 2015). Therefore, our finding that JAK inhibitors induce autophagy in both solid tumors and MPN cells, which then maintain residual disease potentially through the hydrolysis of phospholipids, may offer an explanation of why this class of inhibitors has not been able to eliminate drug-tolerant cancer cells and effect durable responses.

Combination therapies come with the increased risk of side effects. Notably, CYT387, MK2206, and varespladib have all been tested in human clinical trials, and their maximum tolerated doses have been established; the challenge ahead will be to develop optimal dosing schedules that balance target engagement with side effects. However, most small molecule inhibitors have favorable toxicity profiles, and metabolic targets would be non-cross-resistant and predicted to have different side effects that are not overlapping. The experience with infectious diseases highlights the importance of combinations to achieve rapid efficient cancer suppression; i.e., HAART (highly active anti-retroviral therapy) in HIV, which is routinely used to produce durable clinical responses and prevent the emergence of resistance. Polytherapy in cancer is similarly justified and achievable, and here we outline the molecular roadmap for interdicting signaling and metabolism to override treatment-induced autophagy.

\section{Materials and methods}

Cell lines

ACHN, Caki-1, RCC10, SN12C, TK-10, U031, 786-0, UKE-1, SET-2, and HEL were used in this study and were obtained from American Type Culture Collection. ATG5 $5^{+/+}$and ATG5 MEFs were a kind gift from Jay Debnath (University of California at San Francisco). Cell lines were maintained in Dulbecco's modified Eagle's medium (DMEM) supplemented with $10 \%$ fetal bovine serum (FBS) at $37^{\circ} \mathrm{C}$ in a $5 \% \mathrm{CO}_{2}$ incubator.

\section{Patient tumor ex vivo organotypic culture}

Tumor tissue samples were collected at the time of surgical removal from consented patients and transported in IMEM + FBS + PS. The tissue was sliced into thin sections using a surgical knife. Sections were cultured on an organotypic insert (EMD, PICMORG50) for $24 \mathrm{~h}$ in IMEM, 10\% FBS, 1\% PS, and $50 \mu \mathrm{g} /$ $\mathrm{mL}$ holo-transferrin with drug. A section of each tumor was immediately fixed in $10 \%$ buffered formalin to confirm tissue viability. After culture, treated tissue sections were fixed in $10 \%$ buffered formalin and embedded in paraffin. Paraffin-embedded tumors were evaluated for morphology (H\&E) and immunofluorescent signaling.

\section{Cell viability and apoptosis analysis}

Cell viability assays were performed by plating $3 \times 10^{3}$ cells per well in 24-well plates in triplicate and treating them the following day with the indicated agents. The experiment was continued for $5 \mathrm{~d}$, and then the cells were fixed using $4 \%$ formaldehyde and stained for $1 \mathrm{~h}$ with Syto60. Fluorescence was measured and quantified, and photographs were obtained using a LiCor Odyssey infrared imager. The effect of CYT387, MK2206, and the CYT387 +MK2206 combination on cell number was assessed as fold of DMSO-treated control cells. Experimental results are the average of at least three independent experiments. Apoptosis was determined using caspase 3/7 Glo assay kit (Promega) following the manufacturer's instructions. Briefly, 2000 cells per well were plated in 96-well plates and cultured for $72 \mathrm{~h}$. Cells were treated with CYT387, MK2206, and the combination of CYT387 and MK2206 for $72 \mathrm{~h}$, and then $100 \mu \mathrm{L}$ of reagent was added to each well and incubated for $30 \mathrm{~min}$ at room temperature. Caspase 3/ 7 activity was measured using a luminometer. Luminescence values were normalized by cell numbers. The effect of CYT387, MK2206, and the CYT387+MK2206 combination on caspase 3/ 7 activation was assessed as fold of DMSO-treated control cells. 


\section{High-content imaging}

A seven-point dilution series of 116 small molecule inhibitors covering a $1000 \times$ concentration range was plated into three 384well plates using the EP Motion automated dispensing system. Control wells with equal volumes of DMSO were included as negative controls. ACHN cells were grown, trypsinized, counted, and plated directly into warm drug plates using a Multidrop Combi dispenser. Plates were incubated for $72 \mathrm{~h}$ and subsequently imaged on an Olympus ScanR Platform at 10× magnification, performing four images per well in 384-well plates. Singlecell nuclear and cytoplasmic fluorescent intensities were calculated using the Olympus ScanR analysis software: The DAPI-positive region of each cell was used as a boundary to quantitate nucleus counts for analysis of cell growth, and integrated nuclear DNA staining intensity was used for cell cycle analysis. A 10-pixel extension of the nuclear region (and not including the nuclear region) was used to quantitate cytoplasmic signal of immunofluorescent staining of p62 protein and phosphorylation of S6. The mean signal intensity of each marker in all cells per well was used as the metric for cytoplasmic marker expression (average intensity of pS6 and p62). Unsupervised hierarchical clustering was used to identify compounds that produced similar pS6 and p62 dose response phenotypes after treatment.

\section{Western blotting}

Cells were plated in six-well dishes and treated the following day with the indicated agents. Treatments were for $24 \mathrm{~h}$, after which cells were washed with ice-cold PBS and lysed with RIPA buffer (Sigma). Phosphatase inhibitor cocktail set II and protease inhibitor cocktail set III (EMD Millipore) were added at the time of lysis. Lysates were centrifuged at $15,000 \mathrm{~g}$ for $10 \mathrm{~min}$ at $4^{\circ} \mathrm{C}$. Protein concentrations were calculated based on a BCA assay-generated (Thermo Scientific) standard curve. Proteins were resolved using the NuPAGE Novex minigel system on $4 \%-12 \%$ Bis-Tris gels (Invitrogen). For Western blotting, equal amounts of cell lysates (15-20 $\mu \mathrm{g}$ of protein) were resolved with SDS-PAGE and transferred to membranes. The membrane was probed with primary antibodies, washed, and then incubated with corresponding fluorescent secondary antibodies and washed. The fluorescent signal was captured using a LI-COR Odyssey imaging system, and fluorescent intensity was quantified using the Odyssey software where indicated. The following antibodies were used for Western blots: p-S6 (S240/244), S6, LC3B, p-Akt(S473), p-Akt(T308), Akt, and cleaved caspase 3 from Cell Signaling Technologies, and pStat3 (Y705), Stat3, and $\beta$-actin (AC15) from Abcam. Ki67 (Dako) and cleaved caspase 3 (Cell Signaling Technologies) were used for immunohistochemistry. MK2206 and CYT387 for in vitro and in vivo use were purchased from LC Labs and ChemieTek, respectively. BX795 and GDC0941 were purchased from Sigma.

\section{In vivo xenograft studies}

Six-week-old mice were used for human RCC xenografts. For both ACHN and SN12C cell lines, $2 \times 10^{6}$ cells were diluted in $50 \mu \mathrm{L}$ of PBS and $50 \mu \mathrm{L}$ of Matrigel (BD Biosciences) and injected subcutaneously into the right and left flanks of each mouse.

Tumors were monitored until they reached an average size of $50-80 \mathrm{~mm}^{3}$ ( 2 wk), at which point treatments were begun. CYT387 (50 mg/kg per day) was administered by oral gavage $5 \mathrm{~d}$ per week. MK2206 (60 mg/kg per day) was administered by oral gavage 2-3 d per week. CYT387 was dissolved in NMP/Captisol (Cydex), and MK2206 was dissolved in Captisol (Cydex). Tumors and mouse weights were measured twice weekly. At least six to eight mice per treatment group were included. All mice were eu- thanized using $\mathrm{CO}_{2}$ inhalation followed by cervical dislocation per institutional guidelines at Oregon Health and Science University (OHSU). Experiments were approved by the Institutional Animal Care and Use Committee at OHSU.

\section{Phosphoproteomics screen and data analysis}

Enriched phosphopeptides were digested with trypsin and analyzed by MS following the published Cell Signaling Technology protocol (Rush et al. 2005; Moritz et al. 2010; Zhuang et al. 2013).

\section{MS data analysis}

MS raw files were analyzed via MaxQuant version 1.5.3.30 (Cox and Mann 2008), and MS/MS fragmentation spectra were searched using Andromeda (Cox et al. 2011) against human canonical and isoform sequences in Swiss-Prot (downloaded in September 2016 from http://uniprot.org; Apweiler et al. 2004). Quantitative phosphopeptide data were $\log _{10}$ transformed, and missing data were imputed before applying quantile normalization as described previously (Drake et al. 2016). Quantitative data are in Supplemental Tables 3-8. Hierarchical clustering was performed with the Cluster 3.0 program (Eisen et al. 1998) using distance that was based on the Pearson correlation and applying pairwise average linkage analysis. Java Treeview was used to visualize clustering results (Saldanha 2004).

\section{KSEA}

KSEA was performed as described previously (Drake et al. 2012). Briefly, the phosphopeptides were rank-ordered by fold change, on average, between CYT387 treatment and control, and the enrichment score was calculated using the Kolmogorov-Smirnov statistic. Permutation analysis was conducted to calculate statistical significance. The normalized enrichment score was calculated by dividing the enrichment score by the average of the absolute values of all enrichment scores from the permutation analysis (Supplementary Tables 9, 10).

\section{DAVID pathway analysis}

To generate an appropriate list for use in DAVID (Huang da et al. $2009 a, b)$, phosphopeptides were initially filtered with a false discovery rate of $<0.20$ (Supplementary Tables 11,12 ). Phosphopeptides that were 1.5 -fold enriched, on average, in either CYT387 treatment or no treatment were selected. Enrichment for a phosphopeptide was reversed if a functional annotation (Hornbeck et al. 2015) indicated protein activity inhibition. To reduce the complexity of this list, if multiple phosphopeptides mapped to a gene, then the most enriched phosphopeptide was selected. The only exception made was if a functional annotation existed for one or more of the phosphopeptides, in which case the most enriched annotated phosphopeptide would be selected. If multiple phosphopeptides mapped to the same gene and had enrichment values that fell into both CYT387 treatment and no treatment, then those phosphopeptides and the corresponding gene were removed from the list to be analyzed. We input into DAVID the genes in the CYT387 treatment enriched group (Supplemental Table 11) to examine KEGG pathways that were more active with CYT387 treatment (Supplemental Table 12).

\section{Phospho-receptor tyrosine kinase (phospho-RTK) array}

The human phospho-RTK array kit was purchased from Cell Signaling Technologies and screened according to the 
manufacturer's protocol, with $150 \mu \mathrm{g}$ of protein being used for each experiment. Signal intensity was calculated using a LICOR Odyssey imaging system, and fluorescent intensity was quantified using the Odyssey software where indicated.

\section{In vitro ${ }^{18} \mathrm{~F}$-FDG uptake assays}

${ }^{18}$ F-FDG was purchased from the radiopharmacy at University of California at San Francisco. SN12C or ACHN cells $\left(5 \times 10^{5}\right.$ cells $)$ were plated and exposed to vehicle or drug for 24 and $48 \mathrm{~h}$, whereupon the cells were incubated with $0.5 \mu \mathrm{Ci}$ of ${ }^{18} \mathrm{~F}$-FDG for $1 \mathrm{~h}$ at $37^{\circ} \mathrm{C}$. The ${ }^{18} \mathrm{~F}$-FDG in the medium and the intracellular ${ }^{18} \mathrm{~F}$-FDG were isolated and counted using a $\gamma$ counter. The intracellular ${ }^{18} \mathrm{~F}$ FDG was expressed as a percentage of the total activity added to cells normalized to the cell number.

\section{Metabolomic profiling of cancer cells}

Metabolomic data and single-reaction monitoring (SRM) transitions were performed as described previously (Camarda et al. 2016) and are in Supplemental Table 13. Briefly, 2 million cells were plated overnight and serum-starved for $2 \mathrm{~h}$ prior to harvesting, after which cells were washed twice with PBS, harvested by scraping, and flash-frozen. For nonpolar metabolomic analyses, flash-frozen cell pellets were extracted in $4 \mathrm{~mL}$ of $2: 1: 1$ chloroform/methanol/PBS with internal standards: $10 \mathrm{nmol}$ of dodecylglycerol and $10 \mathrm{nmol}$ of pentadecanoic acid. Organic and aqueous layers were separated by centrifugation, and the organic layer was extracted. The aqueous layer was acidified with $0.1 \%$ formic acid followed by re-extraction with $2 \mathrm{~mL}$ of chloroform. The second organic layer was combined with the first extract and dried under nitrogen, after which lipids were resuspended in $120 \mu \mathrm{L}$ of chloroform. A 10- $\mu \mathrm{L}$ aliquot was then analyzed by both SRMbased LC-MS/MS or untargeted LC-MS. For polar metabolomic analyses, frozen cell pellets were extracted in $180 \mu \mathrm{L}$ of 40:40:20 acetonitrile/methanol/water with internal standard: $1 \mathrm{nmol}$ of d3 N15-serine. Following vortexing and bath sonication, the polar metabolite fraction (supernatant) was isolated by centrifugation. A $20-\mu \mathrm{L}$ aliquot was then analyzed by both SRM-based LC-MS/ MS or untargeted LC-MS. For the SRM transitions where we monitored the transition of parent masses to the loss of the head group (e.g., loss of phosphocholine from PC), we ascertained the acyl chain specificities from previously described procedures (Long et al. 2011). For phospholipids such as PCs and PEs, we ascertained fatty acid acyl chain composition from phospholipids using a mobile phase containing both ammonium hydroxide and formic acid and monitored the fatty acid fragmentations from $[\mathrm{M} \mathrm{H}+\mathrm{HCO} 2 \mathrm{H}]$ $\mathrm{m} / \mathrm{z}$ at $40 \mathrm{~V}$ collision energy in negative ionization mode. For other phospholipids, such as PAs and PIs, we monitored the fatty acid fragmentations from $[\mathrm{MH}] \mathrm{m} / \mathrm{z}$ at $40 \mathrm{~V}$ collision energy in negative ionization mode in mobile phase containing just ammonium hydroxide. For the lipids that we measured in this study, the designated acyl chains represent the primary fatty acids that were on the lipid backbone. However, this method is less sensitive than monitoring the loss of head group from the phospholipid, and we therefore used SRM transitions for many phospholipids where we monitored for loss of head groups (e.g., PCs, PEs, PSs, PAs, and PIs).

Relative levels of metabolites were quantified by integrating the area under the curve for each metabolite, normalizing to internal standard values, and then normalizing to the average values of the control groups (Louie et al. 2016).

\section{ROS detection}

ROS levels were measured with CellRox deep red (Molecular Probes). Cells were plated in a 96-well clear-bottomed cell culture plate with black sides. After adhering for $24 \mathrm{~h}$, cells were treated

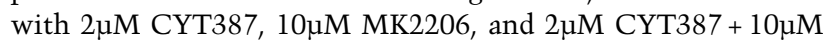
MK2206. The complete medium + drug was removed after $24 \mathrm{~h}$ and replaced with $5 \mu \mathrm{M}$ CellRox deep red in medium. Cells were incubated for $30 \mathrm{~min}$ at $37^{\circ} \mathrm{C}$ and then washed with PBS. Fluorescence signal was detected using a Bioteck Cytation 5 plate reader. Data were analyzed using Prism software.

\section{Cellular respiration}

OCR and ECAR were carried out in a XF96 Seahorse analyzer (Agilent/Seahorse Bioscience,). Cells were plated in the wells of 96-well plates $\left(8 \times 10^{3}\right.$ cells per well; XF96 plates; Seahorse Bioscience) and incubated overnight at $37^{\circ} \mathrm{C}$. The next day, cells were treated with the indicated drugs for $24 \mathrm{~h}$, and then the medium was changed to XF assay medium and loaded with glucose, oligomycin, and 2-DG, respectively, per the manufacturer's recommendation. Similarly, Mito Fuel Flex tests were performed on an XFe96 Bioanalyzer. At $24 \mathrm{~h}$ after treatment, all assays were performed according to the manufacturer's protocols.

\section{Immunohistochemistry}

Immunostaining was performed following deparaffinization and rehydration of slides. Antigen retrieval was performed in a pressure cooker using citrate buffer $(\mathrm{pH}$ 6.0) for $4 \mathrm{~min}$. Nonspecific binding was blocked using Vector mouse IgG blocking serum for $30 \mathrm{~min}$ at room temperature. Samples were incubated at room temperature with rabbit monoclonal antibodies pS6 (Cell Signaling Technologies, 5364), cleaved caspase 3 (Cell Signaling Technologies, 9661), and Ki67 (Dako, M7240). Slides were developed with Vector Immpress rabbit IgG (Vector Laboratories, MP7401) and Vector Immpress mouse IgG (Vector Laboratories, MP7400) for $30 \mathrm{~min}$ at room temperature. Chromogenic detection was performed using Vector Immpact DAB (Vector Laboratories, SK4105) for $3 \mathrm{~min}$. Slides were counterstained with hematoxylin. A 3DHistech MIDI scanner (Perkin Elmer) was used to capture whole-slide digital images with a $20 \times$ objective. Images were converted to MRXS files, and computer graphic analysis was completed using inForm 1.4.0 advanced image analysis software (Perkin Elmer).

\section{Morphological and immunofluorescence evaluation}

H\&E slides of formalin-fixed paraffin-embedded tissue was used to assess the morphological integrity of tumor samples. Once integrity was confirmed, immunofluorescent analysis was performed for p-S6 (1:500; Cell Signaling Technologies), p-AKT (1:200; Cell Signaling Technologies), and LC3B (1:250; Cell Signaling Technologies). Four-micron sections were cut, deparaffinized, and rehydrated. Antigen retrieval was performed using citrate for $4 \mathrm{~min}$ in a pressure cooker. Slides were blocked using $2.5 \%$ normal goat serum for $30 \mathrm{~min}$ and then incubated in primary antibody for $1 \mathrm{~h}$ followed by secondary antibody mouse antirabbit Alexa 488 (1:1000; Molecular Probes) for $30 \mathrm{~min}$. Slides were rinsed in PBS, air dried, and coverslipped using Dako mounting medium with DAPI.

\section{Lipid and mitochondrial staining}

Cells were grown on coverslips and then treated with drug for 24 h. Cells were fixed in $4 \%$ paraformaldehyde for $15 \mathrm{~min}$ and rinsed with PBS. Cells were washed with a $1 \%$ saponin solution for 15 min at room temperature and then washed several times in PBS to remove detergent. Cells were then incubated in Bodipy 
(ThermoFisher, D3922) at a final concentration of $1 \mu \mathrm{M}$ for 10 min. Bodipy was removed, and slides were rinsed with PBS, airdried, and mounted on slides using Dako mounting medium with DAPI.

To detect mitochondrial levels in treated cells, cells were grown on coverslips for $24 \mathrm{~h}$. Mitotracker orange (ThermoFisher, M7511) was diluted in medium with drug at a final concentration of $1 \mathrm{M}$ and incubated overnight. The medium was removed, and cells were fixed with $4 \%$ paraformaldehyde for $15 \mathrm{~min}$. Cells were rinsed twice for $5 \mathrm{~min}$ in PBS and incubated in cold acetone for 10 min at $-20^{\circ} \mathrm{C}$. Acetone was removed, and cells were washed in PBS, air-dried, and mounted on slides with Dako mounting medium with DAPI.

A 3DHistech MIDI scanner (Perkin Elmer) was used to capture whole-slide digital images with a $20 \times$ objective. Images were converted to MRXS files, and computer graphic analysis was completed using inForm 1.4.0 advanced image analysis software (Perkin Elmer).

\section{MDC staining}

Slides were plated on coverslips and allowed to adhere for 24 h. After adherence, cells were treated with drug for $24 \mathrm{~h}$. After treatment, the drug was removed, and cells were washed once in PBS. Cells were labeled with a $50 \mathrm{mM}$ concentration of autofluorescent marker MDC (Sigma) in PBS for $10 \mathrm{~min}$ at $37^{\circ} \mathrm{C}$. Cells were fixed in $4 \%$ formaldehyde for $15 \mathrm{~min}$ at room temperature. Cells were washed twice in PBS for 5 min and mounted on slides using Dako mounting medium with DAPI. Coverslips were sealed with clear nail polish and imaged with a 3 DHistech MIDI scanner as described above.

\section{Statistical analysis}

Mouse tumor size was analyzed by two-way ANOVA with time and drug as factors using Graphpad Prism. Mouse weight during treatment was analyzed by repeated measures of two-way ANOVA with time and drug as factors. A $P$-value of $<0.05$ was considered statistically significant. For immunohistochemistry, $P$-values were calculated using one-way ANOVA with Bonferroni's multiple comparison test $\left(P<0.05\left[{ }^{*}\right], P<0.01\left[{ }^{* *}\right]\right.$, and $P<$ $\left.0.001\left[{ }^{* * *}\right]\right)$. Metabolite fold changes were computed and visualized in Python script using the Openpyxl package (for importing Excel files) and the Matplotlib package (for visualizing fold changes).

\section{Acknowledgments}

We thank Andrew Thorburn, Katherine Ellwood-Yen, and Gary Thomas for helpful discussion; Jay Debnath for the $A T G 5^{+/+}$ and $A T G 5^{-/-}$MEFs; Ernest Frankel, Emek Demir, and Ozgun Babur for computational advice; Steve Kazmierczak for biochemical analysis; Angela Fleischman for clinical input; Mandy Burns and Ashley Sager for administrative support; Moya Costello and Justin Lallo for artwork; the Histopathology Shared Resource for pathology support; the Massively Parallel Sequencing Shared Resource and Integrated Genomics Shared Resource for genomics support; the Bioanalytical Shared Resource/Pharmacokinetic Core for eicosanoid profiling studies; and the Oregon Translational Research and Development Institute (OTRADI) for highthroughput drug screening support. This study was supported by National Institutes of Health (NIH) grants R01 CA169172, P30 CA069533, and P30 CA069533 13S5 through the Oregon Health and Science University-Knight Cancer Institute, the
Hope Foundation (SWOG [Southwest Oncology Group]), the Oregon Translational Research and Development Institute (OTRADI), West Coast Metabolomics Core (WCMC) Pilot, and Kure It Cancer Research (G.V.T.). L.C. is supported by the National Institute of General Medical Sciences of the NIH under award number T32 GM008339. J.M.D. is supported by the Department of Defense (DOD) Prostate Cancer Research Program (W81XWH15-1-0236), Prostate Cancer Foundation Young Investigator Award, and a grant from the New Jersey Health Foundation. V.B.O. is a European Research Council Investigator (LipidArrays) and Royal Society Wolfson Research Merit Award Holder. M.J.E. was supported by the Prostate Cancer Foundation Young Investigator Award, the DOD Prostate Cancer Research Program (W81XWH-15-1-0552), and the National Cancer Institute (R00CA172695 and R01CA176671). C.T. was supported by the DOD Prostate Cancer Research Program (W81XWH-16-1-0435).

\section{References}

Amaravadi RK, Lippincott-Schwartz J, Yin XM, Weiss WA, Takebe N, Timmer W, DiPaola RS, Lotze MT, White E. 2011. Principles and current strategies for targeting autophagy for cancer treatment. Clin Cancer Res 17: 654-666.

Apweiler R, Bairoch A, Wu CH, Barker WC, Boeckmann B, Ferro S, Gasteiger E, Huang H, Lopez R, Magrane M, et al. 2004. UniProt: the Universal Protein knowledgebase. Nucleic Acids Res 32: D115-D119.

Cabodevilla AG, Sanchez-Caballero L, Nintou E, Boiadjieva VG, Picatoste F, Gubern A, Claro E. 2013. Cell survival during complete nutrient deprivation depends on lipid droplet-fueled $\beta$-oxidation of fatty acids. J Biol Chem 288: 27777-27788.

Camarda R, Zhou AY, Kohnz RA, Balakrishnan S, Mahieu C, Anderton B, Eyob H, Kajimura S, Tward A, Krings G, et al. 2016. Inhibition of fatty acid oxidation as a therapy for MYC-overexpressing triple-negative breast cancer. Nat Med 22: $427-432$.

Cecconi F, Levine B. 2008. The role of autophagy in mammalian development: cell makeover rather than cell death. Dev Cell 15: 344-357.

Christofk HR, Vander Heiden MG, Wu N, Asara JM, Cantley LC. 2008. Pyruvate kinase M2 is a phosphotyrosine-binding protein. Nature 452: 181-186.

Chung S, Arrell DK, Faustino RS, Terzic A, Dzeja PP. 2010. Glycolytic network restructuring integral to the energetics of embryonic stem cell cardiac differentiation. J Mol Cell Cardiol 48: $725-734$.

Cox J, Mann M. 2008. MaxQuant enables high peptide identification rates, individualized p.p.b.-range mass accuracies and proteome-wide protein quantification. Nat Biotechnol 26: 1367-1372.

Cox J, Neuhauser N, Michalski A, Scheltema RA, Olsen JV, Mann M. 2011. Andromeda: a peptide search engine integrated into the MaxQuant environment. I Proteome Res 10: 1794-1805.

Dangelmaier C, Manne BK, Liverani E, Jin J, Bray P, Kunapuli SP. 2014. PDK1 selectively phosphorylates Thr(308) on Akt and contributes to human platelet functional responses. Thromb Haemost 111: 508-517.

Debnath J. 2008. Detachment-induced autophagy during anoikis and lumen formation in epithelial acini. Autophagy 4: 351-353.

Dibble CC, Asara JM, Manning BD. 2009. Characterization of Rictor phosphorylation sites reveals direct regulation of mTOR complex 2 by S6K1. Mol Cell Biol 29: 5657-5670. 
Drake JM, Graham NA, Stoyanova T, Sedghi A, Goldstein AS, Cai H, Smith DA, Zhang H, Komisopoulou E, Huang J, et al. 2012. Oncogene-specific activation of tyrosine kinase networks during prostate cancer progression. Proc Natl Acad Sci 109: 1643-1648.

Drake JM, Paull EO, Graham NA, Lee JK, Smith BA, Titz B, Stoyanova T, Faltermeier CM, Uzunangelov V, Carlin DE, et al. 2016. Phosphoproteome integration reveals patient-specific networks in prostate cancer. Cell 166: 1041-1054.

Duvel K, Yecies JL, Menon S, Raman P, Lipovsky AI, Souza AL, Triantafellow E, Ma Q, Gorski R, Cleaver S, et al. 2010. Activation of a metabolic gene regulatory network downstream of mTOR complex 1. Mol Cell 39: 171-183.

Eaton S. 2002. Control of mitochondrial $\beta$-oxidation flux. Prog Lipid Res 41: 197-239.

Eisen MB, Spellman PT, Brown PO, Botstein D. 1998. Cluster analysis and display of genome-wide expression patterns. Proc Nat1 Acad Sci 95: 14863-14868.

Engelman JA, Luo J, Cantley LC. 2006. The evolution of phosphatidylinositol 3-kinases as regulators of growth and metabolism. Nat Rev Genet 7: 606-619.

Finn PF, Dice JF. 2006. Proteolytic and lipolytic responses to starvation. Nutrition 22: 830-844.

Fruman DA, Rommel C. 2014. PI3K and cancer: lessons, challenges and opportunities. Nat Rev Drug Discov 13: 140-156.

Fung C, Lock R, Gao S, Salas E, Debnath J. 2008. Induction of autophagy during extracellular matrix detachment promotes cell survival. Mol Biol Cell 19: 797-806.

Galluzzi L, Pietrocola F, Bravo-San Pedro JM, Amaravadi RK, Baehrecke EH, Cecconi F, Codogno P, Debnath J, Gewirtz DA, Karantza V, et al. 2015. Autophagy in malignant transformation and cancer progression. EMBO J 34: 856-880.

Goldberg SB, Supko JG, Neal JW, Muzikansky A, Digumarthy S, Fidias P, Temel JS, Heist RS, Shaw AT, McCarthy PO, et al. 2012. A phase I study of erlotinib and hydroxychloroquine in advanced non-small-cell lung cancer. I Thorac Oncol 7: 1602-1608.

Gwinn DM, Shackelford DB, Egan DF, Mihaylova MM, Mery A, Vasquez DS, Turk BE, Shaw RJ. 2008. AMPK phosphorylation of raptor mediates a metabolic checkpoint. Mol Cell 30: 214-226.

Hornbeck PV, Zhang B, Murray B, Kornhauser JM, Latham V, Skrzypek E. 2015. PhosphoSitePlus, 2014: mutations, PTMs and recalibrations. Nucleic Acids Res 43: D512-D520.

$\mathrm{Hu} \mathrm{H}$, Juvekar A, Lyssiotis CA, Lien EC, Albeck JG, Oh D, Varma G, Hung YP, Ullas S, Lauring J, et al. 2016. Phosphoinositide 3kinase regulates glycolysis through mobilization of aldolase from the actin cytoskeleton. Cell 164: 433-446.

Huang da W, Sherman BT, Lempicki RA. 2009a. Bioinformatics enrichment tools: paths toward the comprehensive functional analysis of large gene lists. Nucleic Acids Res 37: 1-13.

Huang da W, Sherman BT, Lempicki RA. 2009b. Systematic and integrative analysis of large gene lists using DAVID bioinformatics resources. Nat Protoc 4: 44-57.

Inoki K, Zhu T, Guan KL. 2003. TSC2 mediates cellular energy response to control cell growth and survival. Cell 115: 577-590.

Jeon SM, Chandel NS, Hay N. 2012. AMPK regulates NADPH homeostasis to promote tumour cell survival during energy stress. Nature 485: 661-665.

Joachim J, Jiang M, McKnight NC, Howell M, Tooze SA. 2015. High-throughput screening approaches to identify regulators of mammalian autophagy. Methods 75: 96-104.
Julien LA, Carriere A, Moreau J, Roux PP. 2010. mTORC1-activated S6K1 phosphorylates Rictor on threonine 1135 and regulates mTORC2 signaling. Mol Cell Biol 30: 908-921.

Kerner J, Hoppel C. 2000. Fatty acid import into mitochondria. Biochim Biophys Acta 1486: 1-17.

Kim LC, Cook RS, Chen J. 2017. mTORC1 and mTORC2 in cancer and the tumor microenvironment. Oncogene 36: 2191-2201.

Klionsky DJ, Abdelmohsen K, Abe A, Abedin MJ, Abeliovich H, Acevedo Arozena A, Adachi H, Adams CM, Adams PD, Adeli $\mathrm{K}$, et al. 2016. Guidelines for the use and interpretation of assays for monitoring autophagy (3rd edition). Autophagy 12: $1-222$.

Laplante M, Sabatini DM. 2012. mTOR signaling in growth control and disease. Cell 149: 274-293.

Leonard JT, Rowley JS, Eide CA, Traer E, Hayes-Lattin B, Loriaux M, Spurgeon SE, Druker BJ, Tyner JW, Chang BH. 2016. Targeting BCL-2 and ABL/LYN in Philadelphia chromosome-positive acute lymphoblastic leukemia. Sci Transl Med 8: $354 \mathrm{ra} 114$.

Long JZ, Cisar JS, Milliken D, Niessen S, Wang C, Trauger SA, Siuzdak G, Cravatt BF. 2011. Metabolomics annotates ABHD3 as a physiologic regulator of medium-chain phospholipids. Nat Chem Biol 7: 763-765.

Louie SM, Grossman EA, Crawford LA, Ding L, Camarda R, Huffman TR, Miyamoto DK, Goga A, Weerapana E, Nomura DK. 2016. GSTP1 is a driver of triple-negative breast cancer cell metabolism and pathogenicity. Cell Chem Biol 23: 567-578.

Manning BD, Cantley LC. 2007. AKT/PKB signaling: navigating downstream. Cell 129: 1261-1274.

Manning BD, Tee AR, Logsdon MN, Blenis J, Cantley LC. 2002. Identification of the tuberous sclerosis complex-2 tumor suppressor gene product tuberin as a target of the phosphoinositide 3-kinase/akt pathway. Mol Cell 10: 151-162.

Maxson JE, Gotlib J, Pollyea DA, Fleischman AG, Agarwal A, Eide CA, Bottomly D, Wilmot B, McWeeney SK, Tognon $\mathrm{CE}$, et al. 2013. Oncogenic CSF3R mutations in chronic neutrophilic leukemia and atypical CML. $N$ Engl I Med 368: 1781-1790.

Maxson JE, Abel ML, Wang J, Deng X, Reckel S, Luty SB, Sun H, Gorenstein J, Hughes SB, Bottomly D, et al. 2016. Identification and characterization of tyrosine kinase nonreceptor 2 mutations in leukemia through integration of kinase inhibitor screening and genomic analysis. Cancer Res 76: 127-138.

Mizushima N, Yamamoto A, Hatano M, Kobayashi Y, Kabeya Y, Suzuki K, Tokuhisa T, Ohsumi Y, Yoshimori T. 2001. Dissection of autophagosome formation using Apg5-deficient mouse embryonic stem cells. J Cell Biol 152: 657-668.

Moritz A, Li Y, Guo A, Villen J, Wang Y, MacNeill J, Kornhauser J, Sprott K, Zhou J, Possemato A, et al. 2010. Akt-RSK-S6 kinase signaling networks activated by oncogenic receptor tyrosine kinases. Sci Signal 3: ra64.

Murakami M, Taketomi Y, Miki Y, Sato H, Hirabayashi T, Yamamoto K. 2011. Recent progress in phospholipase A(2) research: from cells to animals to humans. Prog Lipid Res 50: 152-192.

Patel AB, Vellore NA, Deininger MW. 2016. New strategies in myeloproliferative neoplasms: the evolving genetic and therapeutic landscape. Clin Cancer Res 22: 1037-1047.

Pearce EL, Walsh MC, Cejas PJ, Harms GM, Shen H, Wang LS, Jones RG, Choi Y. 2009. Enhancing CD8 T-cell memory by modulating fatty acid metabolism. Nature 460: 103-107.

Rambold AS, Cohen S, Lippincott-Schwartz J. 2015. Fatty acid trafficking in starved cells: regulation by lipid droplet lipolysis, autophagy, mitochondrial fusion dynamics. Dev Cell 32: 678-692. 
Rebecca VW, Amaravadi RK. 2016. Emerging strategies to effectively target autophagy in cancer. Oncogene 35: 1-11.

Rosenfeld MR, Ye X, Supko JG, Desideri S, Grossman SA, Brem S, Mikkelson T, Wang D, Chang YC, Hu J, et al. 2014. A phase I/ II trial of hydroxychloroquine in conjunction with radiation therapy and concurrent and adjuvant temozolomide in patients with newly diagnosed glioblastoma multiforme. Autophagy 10: 1359-1368.

Rosenson RS, Hislop C, Elliott M, Stasiv Y, Goulder M, Waters D. 2010. Effects of varespladib methyl on biomarkers and major cardiovascular events in acute coronary syndrome patients. $J$ Am Coll Cardiol 56: 1079-1088.

Roux PP, Ballif BA, Anjum R, Gygi SP, Blenis J. 2004. Tumor-promoting phorbol esters and activated Ras inactivate the tuberous sclerosis tumor suppressor complex via p90 ribosomal S6 kinase. Proc Natl Acad Sci 101: 13489-13494.

Rush J, Moritz A, Lee KA, Guo A, Goss VL, Spek EJ, Zhang H, Zha XM, Polakiewicz RD, Comb MJ. 2005. Immunoaffinity profiling of tyrosine phosphorylation in cancer cells. Nat Biotechnol 23: 94-101.

Saldanha AJ. 2004. Java Treeview-extensible visualization of microarray data. Bioinformatics 20: 3246-3248.

Sarker D, Ang JE, Baird R, Kristeleit R, Shah K, Moreno V, Clarke PA, Raynaud FI, Levy G, Ware JA, et al. 2015. First-in-human phase I study of pictilisib (GDC-0941), a potent pan-class I phosphatidylinositol-3-kinase (PI3K) inhibitor, in patients with advanced solid tumors. Clin Cancer Res 21: 77-86.

Sehgal AR, Konig H, Johnson DE, Tang D, Amaravadi RK, Boyiadzis M, Lotze MT. 2015. You eat what you are: autophagy inhibition as a therapeutic strategy in leukemia. Leukemia 29: $517-525$

Shanware NP, Bray K, Abraham RT. 2013. The PI3K, metabolic, autophagy networks: interactive partners in cellular health and disease. Annu Rev Pharmacol Toxicol 53: 89-106.

Singh R, Kaushik S, Wang Y, Xiang Y, Novak I, Komatsu M, Tanaka K, Cuervo AM, Czaja MJ. 2009. Autophagy regulates lipid metabolism. Nature 458: 1131-1135.

Slatter DA, Aldrovandi M, O'Connor A, Allen SM, Brasher CJ, Murphy RC, Mecklemann S, Ravi S, Darley-Usmar V, O'Donnell VB. 2016. Mapping the human platelet lipidome reveals cytosolic phospholipase A2 as a regulator of mitochondrial bioenergetics during activation. Cell Metab 23: 930-944.
Sztalryd C, Kimmel AR. 2014. Perilipins: lipid droplet coat proteins adapted for tissue-specific energy storage and utilization, and lipid cytoprotection. Biochimie 96: 96-101.

Tanida I, Minematsu-Ikeguchi N, Ueno T, Kominami E. 2005. Lysosomal turnover, but not a cellular level, of endogenous LC3 is a marker for autophagy. Autophagy 1: 84-91.

Thiam AR, Farese RV Jr, Walther TC. 2013. The biophysics and cell biology of lipid droplets. Nat Rev Mol Cell Biol 14: 775-786.

Toska E, Baselga J. 2016. Pharmacology in the era of targeted therapies: the case of PI3K inhibitors. Clin Cancer Res 22: 2099-2101.

Towers CG, Thorburn A. 2016. Therapeutic targeting of autophagy. EBioMedicine 14: 15-23.

Turcotte S, Chan DA, Sutphin PD, Hay MP, Denny WA, Giaccia AJ. 2008. A molecule targeting VHL-deficient renal cell carcinoma that induces autophagy. Cancer Cell 14: 90-102.

Unger RH, Clark GO, Scherer PE, Orci L. 2010. Lipid homeostasis, lipotoxicity and the metabolic syndrome. Biochim Biophys Acta 1801: 209-214.

Vannucchi AM, Kiladjian JJ, Griesshammer M, Masszi T, Durrant S, Passamonti F, Harrison CN, Pane F, Zachee P, Mesa $\mathrm{R}$, et al. 2015. Ruxolitinib versus standard therapy for the treatment of polycythemia vera. N Engl J Med 372: 426-435.

Verstovsek S, Mesa RA, Gotlib J, Levy RS, Gupta V, DiPersio JF, Catalano JV, Deininger M, Miller C, Silver RT, et al. 2012. A double-blind, placebo-controlled trial of ruxolitinib for myelofibrosis. N Engl I Med 366: 799-807.

Winton EF, Kota V. 2017. Momelotinib in myelofibrosis: JAK1/2 inhibitor with a role in treating and understanding the anemia. Future Oncol 13: 395-407.

Yap TA, Yan L, Patnaik A, Fearen I, Olmos D, Papadopoulos K, Baird RD, Delgado L, Taylor A, Lupinacci L, et al. 2011. First-in-man clinical trial of the oral pan-AKT inhibitor MK2206 in patients with advanced solid tumors. J Clin Oncol 29: 4688-4695.

Yecies JL, Manning BD. 2011. Transcriptional control of cellular metabolism by mTOR signaling. Cancer Res 71: 2815-2820.

Zhuang G, Yu K, Jiang Z, Chung A, Yao J, Ha C, Toy K, Soriano R, Haley B, Blackwood E, et al. 2013. Phosphoproteomic analysis implicates the mTORC2-FoxO1 axis in VEGF signaling and feedback activation of receptor tyrosine kinases. Sci Signal 6: ra25. 


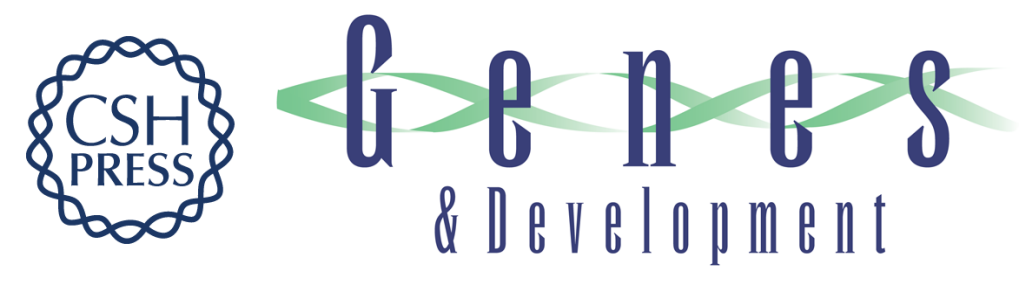

\section{Metabolic reprogramming ensures cancer cell survival despite oncogenic signaling blockade}

Hui-wen Lue, Jennifer Podolak, Kevin Kolahi, et al.

Genes Dev. 2017, 31: originally published online November 14, 2017

Access the most recent version at doi:10.1101/gad.305292.117

\section{Supplemental http://genesdev.cshlp.org/content/suppl/2017/11/14/gad.305292.117.DC1 Material}

References This article cites 73 articles, 20 of which can be accessed free at: http://genesdev.cshlp.org/content/31/20/2067.full.html\#ref-list-1

Creative This article is distributed exclusively by Cold Spring Harbor Laboratory Press for the first Commons six months after the full-issue publication date (see

License http://genesdev.cshlp.org/site/misc/terms.xhtml). After six months, it is available under a Creative Commons License (Attribution-NonCommercial 4.0 International), as described at http://creativecommons.org/licenses/by-nc/4.0/.

Email Alerting Receive free email alerts when new articles cite this article - sign up in the box at the top Service right corner of the article or click here.

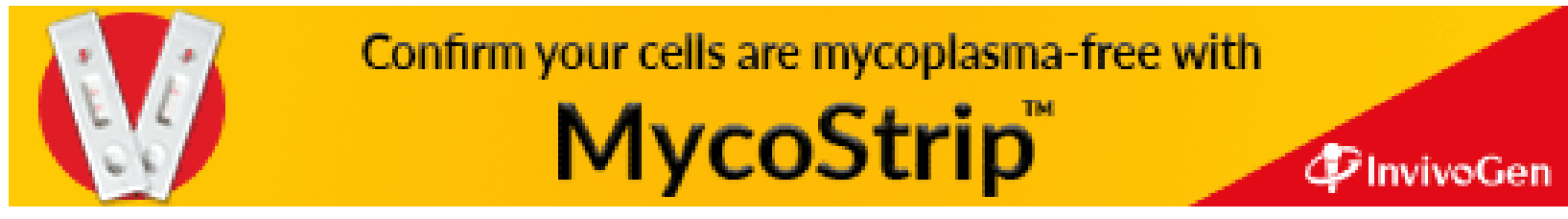

